\title{
Spectroscopy of proton-unbound nuclei by tracking their decay products in-flight: One- and two- proton decays of ${ }^{15} \mathrm{~F},{ }^{16} \mathrm{Ne}$, and ${ }^{19} \mathrm{Na}$
}

I. Mukha,,${ }^{1,2,3,{ }^{*}}$ K. Sümmerer, ${ }^{1}$ L. Acosta, ${ }^{4}$ M. A. G. Alvarez,${ }^{5}$ E. Casarejos, ${ }^{6,7}$ A. Chatillon, ${ }^{1}$ D. Cortina-Gil, ${ }^{6}$ I. A. Egorova, ${ }^{8}$ J. M. Espino, ${ }^{5}$ A. Fomichev, ${ }^{9}$ J. E. García-Ramos, ${ }^{4}$ H. Geissel, ${ }^{1}$ J. Gómez-Camacho, ${ }^{5}$ L. Grigorenko, ${ }^{1,9}$ J. Hofmann, ${ }^{1}$ O. Kiselev, ${ }^{1,10}$ A. Korsheninnikov, ${ }^{3}$ N. Kurz, ${ }^{1}$ Yu. A. Litvinov, ${ }^{1,11}$ E. Litvinova, ${ }^{1,12}$ I. Martel, ${ }^{4}$ C. Nociforo, ${ }^{1}$ W. Ott, ${ }^{1}$ M. Pfützner, ${ }^{13}$ C. Rodríguez-Tajes, ${ }^{6,14}$ E. Roeckl, ${ }^{1}$ M. Stanoiu, ${ }^{1,15}$ N. K. Timofeyuk, ${ }^{16}$ H. Weick, ${ }^{1}$ and P. J. Woods ${ }^{17}$

${ }^{1}$ GSI Helmholtzzentrum für Schwerionenforschung, D-64291 Darmstadt, Germany

${ }^{2}$ CSIC - IFIC Universidad de Valencia, E-46071 Valencia, Spain

${ }^{3}$ RRC “Kurchatov Institute," RU-123184 Moscow, Russia

${ }^{4}$ Department of Applied Physics, Universidad de Huelva, E-21071 Huelva, Spain

${ }^{5}$ Universidad de Sevilla, E-41012 Seville, Spain

${ }^{6}$ Universidade de Santiago de Compostela, E-15782 Santiago de Compostela, Spain

${ }^{7}$ Universidade de Vigo, E-36310, Vigo, Spain

${ }^{8}$ Bogolubov Laboratory of Theoretical Physics, JINR, RU-141980 Dubna, Russia

${ }^{9}$ Joint Institute for Nuclear Research, RU-141980 Dubna, Russia

${ }^{10}$ Paul Scherrer Institut, $\mathrm{CH}-5232$ Villigen, Switzerland

${ }^{11}$ Max-Planck Institut für Kernphysik, D-69117 Heidelberg, Germany

${ }^{12}$ Institut für Theoretische Physik, Goethe Universität, D-60438 Frankfurt am Main, Germany

${ }^{13}$ IEP, Warsaw University, PL-00681 Warszawa, Poland

${ }^{14}$ Centro de Láseres Pulsados (CLPU), E-37008 Salamanca, Spain

${ }^{15}$ IFIN-HH, Post Office Box MG-6, Bucharest, Romania

${ }^{16}$ Department of Physics, School of Electronics Physical Sciences, University of Surrey, Guildford, GU2 7XH, United Kingdom

${ }^{17}$ University of Edinburgh, EH1 1HT Edinburgh, United Kingdom

(Received 3 September 2010; published 29 November 2010)

\begin{abstract}
A powerful method of investigating proton-unbound nuclear states by tracking their decay products in flight is discussed in detail. To verify the method, four known levels in ${ }^{15} \mathrm{~F},{ }^{16} \mathrm{Ne}$, and ${ }^{19} \mathrm{Na}$ were investigated by measuring the angular correlations between protons and the respective heavy-ion fragments stemming from the precursor decays in flight. The parent nuclei of interest were produced in nuclear reactions of one-neutron removal from ${ }^{17} \mathrm{Ne}$ and ${ }^{20} \mathrm{Mg}$ projectiles at energies of $410-450 \mathrm{~A} \mathrm{MeV}$. The trajectories of the respective decay products, ${ }^{14} \mathrm{O}+\mathrm{p}+\mathrm{p}$ and ${ }^{18} \mathrm{Ne}+\mathrm{p}+\mathrm{p}$, were measured by applying a tracking technique with microstrip detectors. These data were used to reconstruct the angular correlations of the fragments, which provided information on energies and widths of the parent states. In addition for reproducing properties of known states, evidence for hitherto unknown excited states in ${ }^{15} \mathrm{~F}$ and ${ }^{16} \mathrm{Ne}$ was found. This tracking technique has an advantage in studies of exotic nuclei beyond the proton drip line measuring the resonance energies and widths with a high precision although by using low-intensity beams and very thick targets.
\end{abstract}

DOI: 10.1103/PhysRevC.82.054315

PACS number(s): 21.10.-k, 23.50.+z, 21.45.-v, 27.20.+n

\section{NUCLEAR STRUCTURE BEYOND THE PROTON DRIP LINE}

Nuclear structure beyond the proton drip line, where nuclei are unbound and exist only as resonances in the continuum, is still a rather unexplored topic despite substantial experimental advances made lately. In particular, the recently discovered phenomenon of two-proton $(2 \mathrm{p}$ ) radioactivity, the spontaneous break-up of an atomic nucleus by the emission of two protons, displays unexpectedly long half-lives for all reported $2 p$ precursors, ${ }^{45} \mathrm{Fe},{ }^{54} \mathrm{Zn},{ }^{19} \mathrm{Mg}$, and ${ }^{94 m} \mathrm{Ag}$ [1-4] in comparison with the predictions of a quasiclassical estimate of the emission of ${ }^{2} \mathrm{He}$. A quantum-mechanical theory of $2 p$ radioactivity based on a three-body model of nuclear structure of a $2 p$-decay precursor (which assumes a configuration $p+p+$ "core") [5-7]

*I.Mukha@gsi.de explains such long half-lives as the result of a decay retardation due to the higher three-body centrifugal barrier in the parent nucleus. The theory predicts the regular occurrence of longlived $2 p$ precursors in the region beyond the proton drip line. Calculating correctly the properties of $2 p$-unbound nuclei with a three-body model may also be of interest for nuclear astrophysics. The inverse reaction to $2 p$ decay, radiative $2 p$ capture, may play an important role in the synthesis of heavy elements in the universe, by possibly bridging some "waiting points" in the $r p$ process (see, e.g., [8-10]). Measurements of $2 p$ decays are so far the only way of studying radiative $2 \mathrm{p}$ capture.

In general, one expects one-proton (1p) or $2 p$ decaying resonances in light nuclei to be very broad due to small Coulomb barriers. For example, the ground states (g.s.) of ${ }^{15} \mathrm{~F}$ or ${ }^{10,11} \mathrm{~N}$ are seen as broad $s$-wave proton resonances (see, e.g., Refs. [11-15]). Only a few excited states of such nuclei located beyond the proton drip line are known. Their widths are usually of the order of hundreds of $\mathrm{keV}$. 
However, some proton-unbound nuclei whose structure is more complicated than a single-particle configuration (i.e., a proton orbit around an inert nuclear core) may exist as very long-lived resonances due to high few-body centrifugal barriers and/or small 1p spectroscopic factors. In particular, the measured $1 p$ and $2 p$ decays of the recently found excited states in ${ }^{15} \mathrm{~F}$ and ${ }^{16} \mathrm{Ne}$ [11] gave evidence for relatively stable nuclear configurations beyond the proton drip line. The observed states have much smaller widths compared to the respective predictions of models assuming single-particle configurations of parent states. Their structure may be ascribed to particlehole configurations where protons are coupled to core-excited states. Then 1p-decay branches of such configurations into the excited-core states are larger than those into the respective g.s.'s [16,17]. The excited-core daughters are, in turn, open to $1 \mathrm{p}$ decays, and therefore the corresponding final states comprise three particles. Such a phenomenon is expected to be general for nuclei beyond the proton drip line where $1 \mathrm{p}$ and $2 \mathrm{p}$ thresholds are very low.

The time scale of nuclear decays by proton emission accessible in experiments spans from $10^{-2} \mathrm{~s}$ (for the longer lifetimes, weak decays prevail) to $10^{-21} \mathrm{~s}$ (for the shorter lifetimes, continuum dynamics are important). Such a broad range can be accessed by different experimental techniques. In the case of nuclear $1 p$ and $2 p$ decays with lifetimes larger than a few microseconds, one can implant the radioactive atoms and subsequently detect their decay. For much shorter half lives, the conventional in-flight-decay method aims at detecting all fragments of a proton precursor in missing-mass or invariant-mass measurements. A novel experimental technique for measuring in-flight decays of proton-unbound nuclei with lifetimes in the time range of $10^{-7}-10^{-12} \mathrm{~s}$ was suggested in Ref. [18] and discussed in Refs. [7,19,20]. In such a measurement, the trajectories of all decay products are tracked and the decay vertices as well as the angular correlations of the decay products can be deduced from the measured trajectories. For the first time in studies of radioactivity, we performed an in-flight-decay experiment by the tracking technique with microstrip detectors. Some results of the experiment were published in previous articles. The observations of ${ }^{19} \mathrm{Mg}$ and its $2 \mathrm{p}$ radioactivity [3], proton-proton correlations from $2 \mathrm{p}$ decays of ${ }^{19} \mathrm{Mg}$ and ${ }^{16} \mathrm{Ne}$ [21], and new resonances in ${ }^{15} \mathrm{~F}$ populated by $1 \mathrm{p}$ decay of excited states in ${ }^{16} \mathrm{Ne}$ [11] were reported. Due to space limitations, some important details of the experimental technique and methods of analysis remained unexplained. An understanding of these details is crucial for future applications of the presented method in studies of nucleari specata beyond the proton drip line.

In a series of two articles, we intend to present more experimental details concerning the previously published results and to provide new data on unobserved nuclear states. In the present article, the first of the series, we describe the tracking technique in more detail and verify it by reproducing the properties of previously known proton-unbound states in the isotopes ${ }^{15} \mathrm{~F},{ }^{16} \mathrm{Ne}$, and ${ }^{19} \mathrm{Na}$. The article is structured in the following way. In Sec. II, the experimental setup and the tracking procedure of charged particles with microstrip detectors are described. The method of identification of a 2 p-precursor state of interest, measuring its decay energy and width by using angular correlations of its decay products is discussed in Sec. III. A comparison of selected cases of $1 p$ and $2 p$ decays with literature data is given in Sec. IV. Spectroscopic results on previously unknown proton-unbound states in the isotopes ${ }^{15} \mathrm{~F}$ and ${ }^{16} \mathrm{Ne}$ are presented in Sec. V. Finally, the advantages of the tracking technique in studies of nuclear structure beyond the proton drip line are presented in Sec. VI. More experimental details of applying the tracking method to lifetime measurements in the nanosecond to femtosecond regime, as pioneered for the case of ${ }^{19} \mathrm{Mg}$ [3], will form the subject of a forthcoming article.

\section{EXPERIMENTAL METHOD}

The experiment was performed by using a $591 A \mathrm{MeV}$ beam of ${ }^{24} \mathrm{Mg}$ accelerated by the SIS facility at GSI, Darmstadt. A sketch of the experimental setup is presented in Fig. 1 where the upper panel shows the general layout and the lower panel provides a closeup view of the tracking detectors. Radioactive beams of ${ }^{20} \mathrm{Mg}$ and ${ }^{17} \mathrm{Ne}$ were produced at the Projectile Fragment Separator (FRS) [22] with average intensities of 400 and 800 ions s $^{-1}$ and energies of 450 and $410 \mathrm{~A} \mathrm{MeV}$, respectively. In total, about $2.2 \times 10^{7}$ of ions of ${ }^{20} \mathrm{Mg}$ and $9.1 \times 10^{6}$ of ${ }^{17} \mathrm{Ne}$ were produced. The secondary reactions $\left({ }^{20} \mathrm{Mg},{ }^{19} \mathrm{Mg}\right)$ and $\left({ }^{17} \mathrm{Ne},{ }^{16} \mathrm{Ne}\right)$ occurred at the midplane of FRS in a $2 \mathrm{~g} / \mathrm{cm}^{2}{ }^{9} \mathrm{Be}$ reaction target. Special ion-optical settings for FRS were applied, the first FRS half being tuned in an achromatic mode using a wedge-shaped degrader at the first focal plane F1, while its second half was set for identification of the heavy ions (HI), in particular ${ }^{17} \mathrm{Ne}$ and ${ }^{14} \mathrm{O}$, with high acceptance in angle and momentum.

At the FRS midplane, a $6 \times 6 \mathrm{~cm}^{2}$ double-sided Si detector (DSSD) with $32 \times 32$ strips and a $20 \times 20 \mathrm{~cm}^{2}$ multiwire chamber were used upstream of the reaction target to track
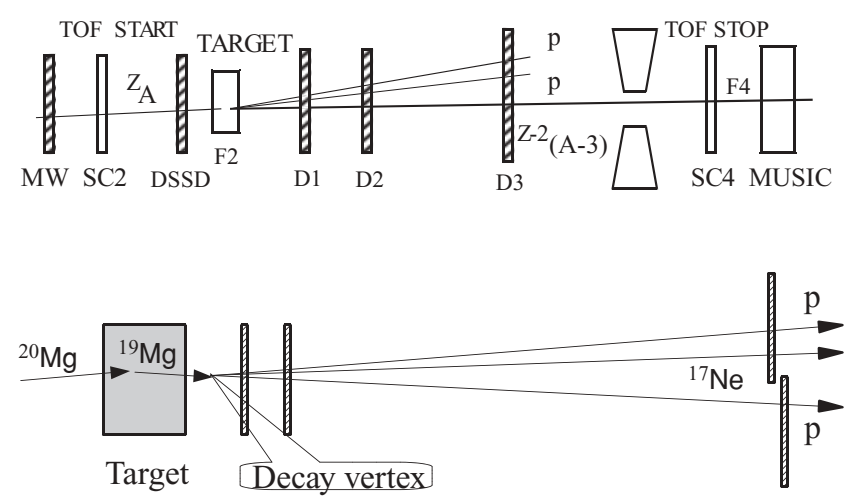

FIG. 1. Upper panel: Sketch of the experimental setup located at the FRS midplane. The secondary beams were tracked by a DSSD and a multiwire chamber (MW). The reaction products were formed in fragmentation reactions in the reaction target located at the FRS focus F2. Outgoing protons and heavy ions were tracked in triple coincidences $\mathrm{HI}+\mathrm{p}+\mathrm{p}$ by three planes of silicon micro-strip detectors (D1-D3). The heavy ions were identified at the foci F3 and F4 by magnetic-rigidity, time-of-flight (TOF), and energy loss measurements with the scintillator detector Sc4 at F4. Lower panel: Illustration of tracking $2 p$ decays of ${ }^{19} \mathrm{Mg}$ with the three planes of microstrip detectors. 


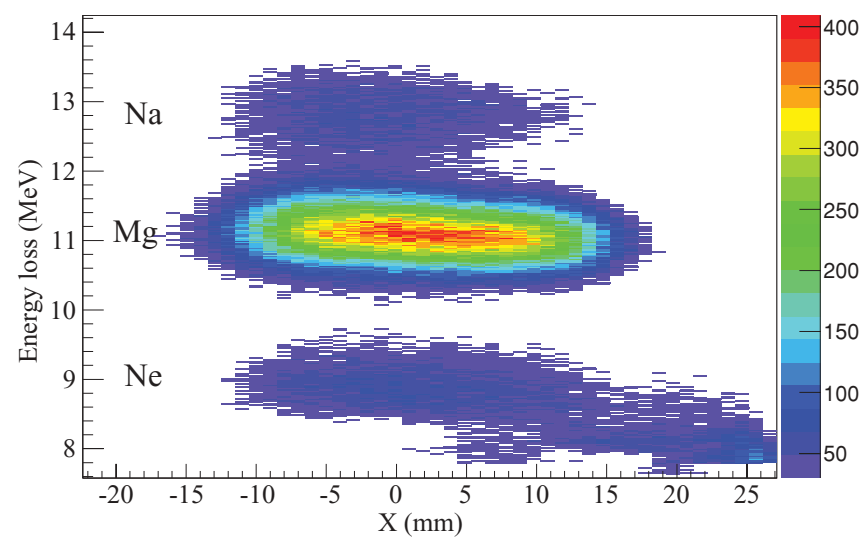

FIG. 2. (Color online) Transverse spatial profile of ions versus their energy loss of a secondary beam detected in front of the FRS reaction target by the DSSD detector. The ion-optical settings of the first half of FRS are optimized for a $410 \mathrm{~A} \mathrm{MeV}$ beam of ${ }^{20} \mathrm{Mg}$.

the incoming ${ }^{20} \mathrm{Mg}\left({ }^{17} \mathrm{Ne}\right)$ projectiles. As an example, the identification plot used to identify ${ }^{20} \mathrm{Mg}$ projectiles detected by DSSD is shown in Fig. 2. Downstream from the reaction target, a microstrip detector array [23], developed on the basis of the tracker of the AMSO2 project [24], was positioned. It consisted of four large-area $\left(7 \times 4 \mathrm{~cm}^{2}\right)$ double-sided silicon microstrip detectors (MSD) with a pitch of $0.1 \mathrm{~mm}$ on both sides, covering an opening angle of $\sim 150 \mathrm{mrad}$ around the beam direction. They were used to measure energy losses and positions of coincident hits of 2p's and a heavy fragment, thus allowing us to reconstruct all decay-product trajectories and derive the coordinates of the reaction vertex and the angular $\mathrm{p}-\mathrm{p}$ and proton-HI correlations.

In the second half of FRS, the heavy-ion decay products like ${ }^{17} \mathrm{Ne}$ or ${ }^{14} \mathrm{O}$ were unambiguously identified by their times of flight, their magnetic rigidities, and their energy losses measured with position-sensitive scintillator detectors. As an example, an HI identification plot is shown in Fig. 3 where the fragment atomic numbers $Z$ are plotted versus their mass $(A)$ over-charge $(Q)$ ratios, AoQ (at our energies, ions were fully

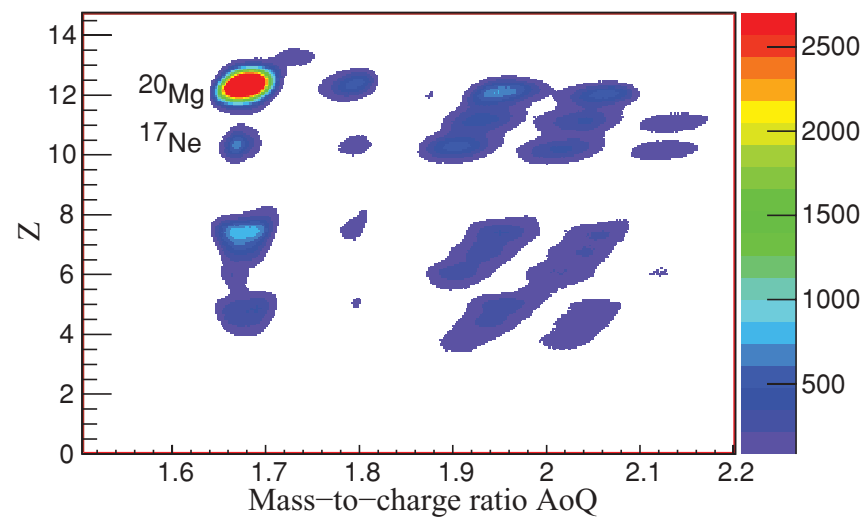

FIG. 3. (Color online) Identification plot for ions detected at the FRS focal planes F2 and F4. The ion-optical setting was optimized for the transmission of ${ }^{17} \mathrm{Ne}$ ions produced in the reaction target by a $410 \mathrm{~A} \mathrm{MeV}$ beam of ${ }^{20} \mathrm{Mg}$. The $Y$ axis represents the element number, $Z$, the $X$ axis displays the mass-to-charge ratio, AoQ.

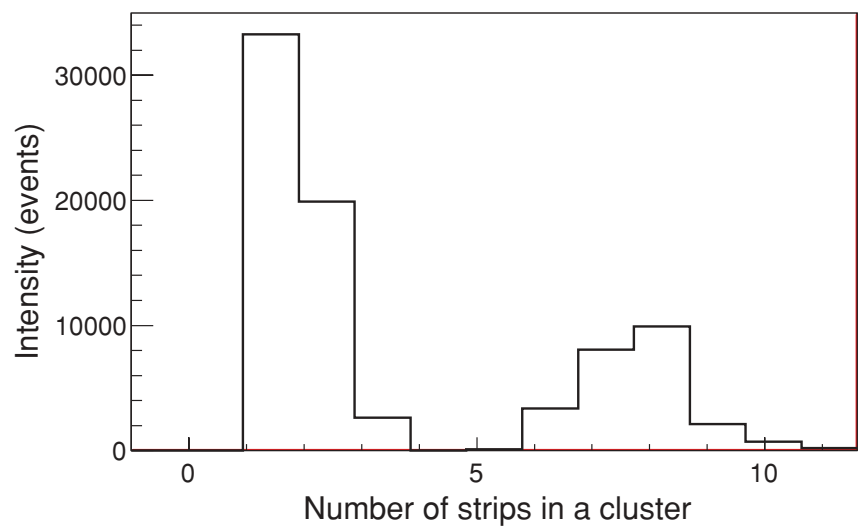

FIG. 4. Typical numbers of strips fired in microstrip detectors by $400 \mathrm{~A} \mathrm{MeV}$ protons (left) and ${ }^{17} \mathrm{Ne}$ ions (right).

stripped and $Z=Q)$. Nuclear mass $(A)$-over-charge $(Q)$ ratios, AoQ were determined by a standard method [22] from the measured times of flight and positions at the focal planes (which allowed to calculate the respective magnetic rigidities) by taking into account the full tracking information at the F2 and F4 focal planes of FRS. As one may see in Fig. 3, the AoQ values of secondary-beam ions ${ }^{20} \mathrm{Mg}$ and fragments ${ }^{17} \mathrm{Ne}$ are very close, which demonstrates the purity of the ${ }^{20} \mathrm{Mg}$ secondary beam obtained by the achromatic tuning of the ion optics in the first half of FRS. The ${ }^{20} \mathrm{Mg}$ isotopes constituted more than $90 \%$ of the secondary-beam ions registered at F2. The small contamination consisted mainly of ${ }^{17} \mathrm{Ne}$, even smaller admixtures of ${ }^{21} \mathrm{Mg},{ }^{23} \mathrm{Al}$ and so on were also present.

Fragment tracking was based on the response of the doublesided microstrip detectors (MSD, labeled D1, D2, and D3 in Fig. 1) to protons and heavy ions. Due to the capacitive coupling between neighboring strips, in most cases more than one strip shows a finite signal above the strip pedestal. In Fig. 4, the numbers of strips fired in MSD is shown for protons and ${ }^{17} \mathrm{Ne}$ ions.

One can see that protons fire mostly only one strip. Thus their position uncertainty is $\sigma_{x}=100 / \sqrt{12} \approx 30 \mu \mathrm{m}$. The $\mathrm{Ne}(\mathrm{Mg})$ ions typically produce clusters of 7(9) strips, which is due to capacitive coupling between the neighboring strips. In an individual detector, the cluster centroid can be determined with an accuracy of about $14 \mu \mathrm{m}$. The cluster areas are proportional to the energy losses of the impinging ions, a typical example of the energy loss measured by the MSD is shown in Fig. 5.

In the present study, we were mainly interested in observing the coincident event of heavy ions (HI) and 2p's. Conditions for true $\mathrm{HI}+\mathrm{p}+\mathrm{p}$ events were (i) the minimal distance between proton and heavy-ion trajectories was required to be very small, typically less than $150 \mu \mathrm{m}$, and (ii) the difference between the two longitudinal coordinates of the vertices defined by each of the two p-HI pairs (taken from the same $\mathrm{HI}+\mathrm{p}+\mathrm{p}$ event) had to lie within the range defined by the experimental uncertainty of $0.3-1 \mathrm{~mm}$, depending on the detection angle. The achieved angular resolution in fragment tracking was $\sim 1 \mathrm{mrad}$.

The inset in Fig. 5 shows that the proton can be well separated from noise if triple $\mathrm{HI}+\mathrm{p}+\mathrm{p}$ coincidences are 


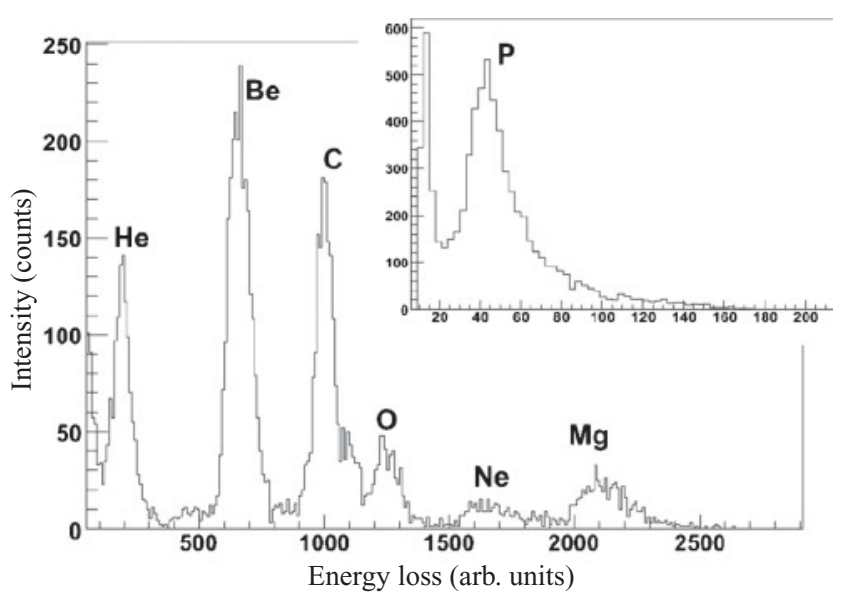

FIG. 5. Typical energy-loss spectrum of different ions derived from the hit-cluster areas on the $X$ side of the microstrip detector [23]. The inset shows the proton energy-loss spectrum derived from coincident triple ${ }^{17} \mathrm{Ne}+\mathrm{p}+\mathrm{p}$ events.

required. The energy-loss spectra for those $\mathrm{HI}+\mathrm{p}+\mathrm{p}$ events are particularly clean, as shown in Fig. 6. Under these conditions, proton signals are well separated from the heavyion ones.

To track ions through the three layers of MSD in the present experiment, it is necessary to determine the offsets of the detectors relative to each other. Such an alignment procedure uses single-ion tracks crossing all three layers of MSD. For each detector, five alignment parameters were determined, namely two spatial $\left(X_{0}, Y_{0}\right)$ and three angular $\left(\theta_{0}^{x}\right.$, $\left.\theta_{0}^{y}, \theta_{0}^{z}\right)$ offsets. The offsets were determined by minimizing the differences $d X$ and $d Y$ between the $X$ and $Y$ coordinates of ${ }^{20} \mathrm{Mg}$ ions in the secondary-target plane as derived from the two alternative trajectories given by the different MSD pairs, D1-D3 and D2-D3 (see Fig. 1). The offsets were changed iteratively until $d X$ and $d Y$ were minimal. The result of this procedure is illustrated in Fig. 7, where the $d Y$ is plotted versus $d X$, the differences between the two alternative coordinates $(X, Y)$ obtained for each ${ }^{20} \mathrm{Mg}$ ion on the secondary-target plane (see Fig. 1). Projecting the $d X-d Y$ distribution on the axes yields full width at half maximum (FWHM) widths

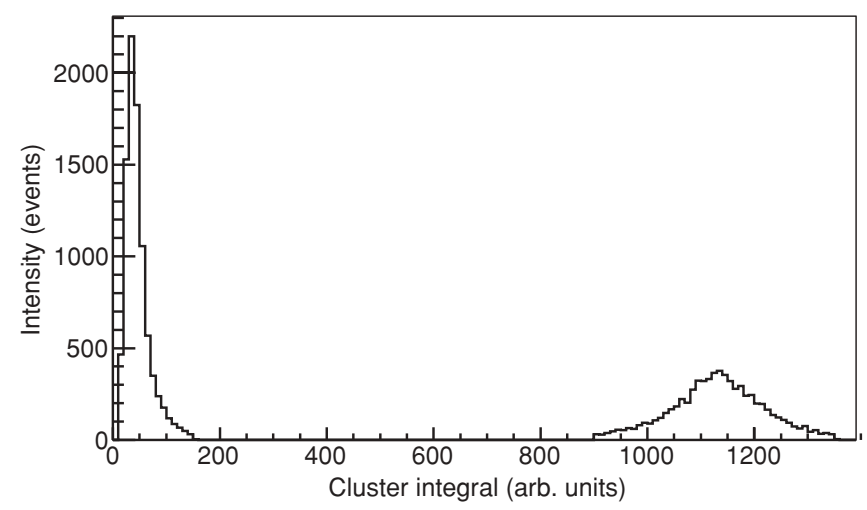

FIG. 6. Distribution of uncorrected cluster areas from the $Y$ side of the microstrip detector derived from triple ${ }^{15} \mathrm{O}+\mathrm{p}+\mathrm{p}$ coincidences.

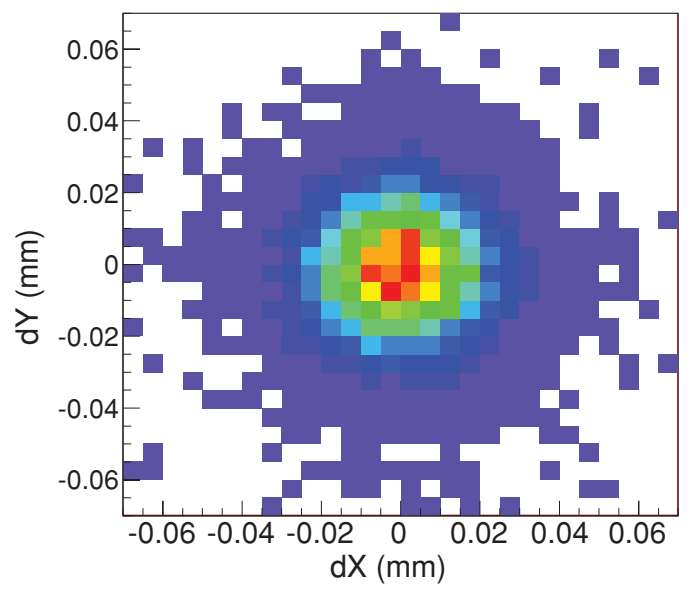

FIG. 7. (Color online) Uncertainties of positions of ${ }^{20} \mathrm{Mg}$ ions at the reaction target in the $(X, Y)$-transverse directions. The uncertainties were evaluated from the differences between two alternative $(X, Y)$-coordinate values of the ${ }^{20} \mathrm{Mg}$ positions at the target derived from two alternative ${ }^{20} \mathrm{Mg}$ trajectories defined by the MSD pairs D1-D3 and D2-D3, see Fig. 1.

of $\approx 10 \mu \mathrm{m}$ in both directions. Though the used procedure of alignment is simplified in comparison with a complete procedure which makes a minimization of all five offsets at the same time (e.g., see the Compact Muon Solenoid (CMS) tracking procedure [25]), the achieved tracking accuracy serves well for our physics case.

Proton-HI vertices are defined as points where the distance between the respective trajectories is minimal. The inclusive distribution of these distances-of-minimal-approach is illustrated in Fig. 8. A three-particle $\mathrm{HI}+\mathrm{p}+\mathrm{p}$ vertex is given by averaging the coordinates of the two two-particle vertices. It is obvious that in $Z$ (beam) direction the uncertainty of the vertex depends on the $2 \mathrm{p}-\mathrm{HI}$ angles (taken from the same $\mathrm{HI}+\mathrm{p}+\mathrm{p}$ event), yielding a larger uncertainty in the case of smaller $\mathrm{p}+\mathrm{HI}$ angles due to the almost parallel movement of the ions. Typical uncertainties range from 0.3 to $1 \mathrm{~mm}$, see the respective histograms in the upper and lower panels of Fig. 9. The uncertainty of the centroid of an $N$-event vertex distribution is smaller than the respective single-event uncertainty by a factor of $1 / \sqrt{N}$. For example, the centroid of the ${ }^{19} \mathrm{Mg}$ $\rightarrow{ }^{17} \mathrm{Ne}+\mathrm{p}+\mathrm{p}$ vertex distribution (about 300 events) can be

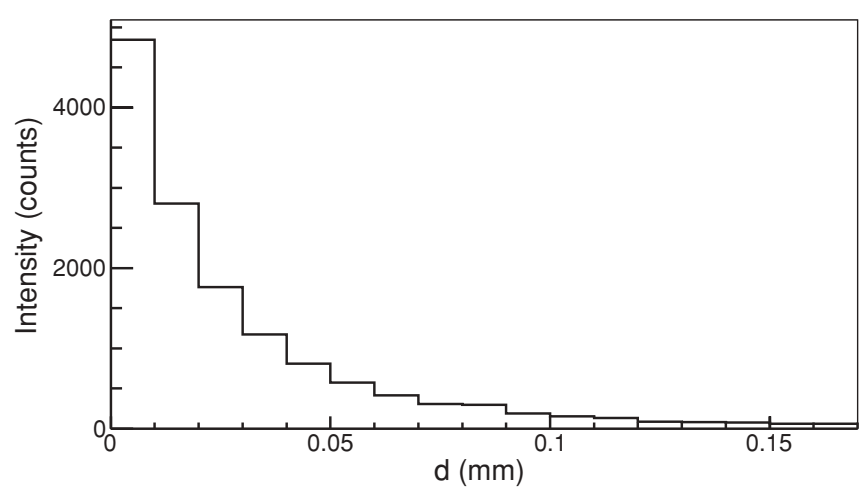

FIG. 8. Distances of the closest approach $d$ of two trajectories of a proton and an ${ }^{17} \mathrm{Ne}$ ion derived from tracked ${ }^{17} \mathrm{Ne}+\mathrm{p}+\mathrm{p}$ events. 

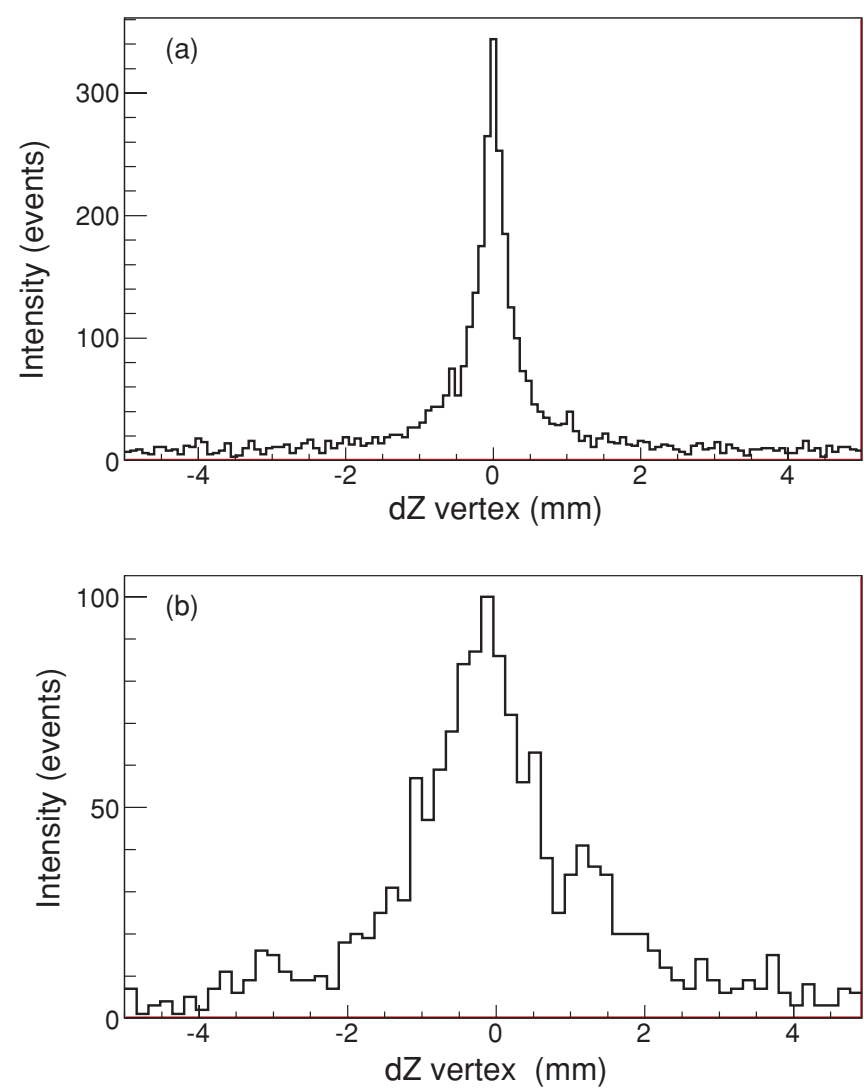

FIG. 9. Spatial differences between two decay vertices along the $Z$ (beam) direction defined for the $\mathrm{p}_{1}{ }^{-17} \mathrm{Ne}$ and $\mathrm{p}_{2}-{ }^{17} \mathrm{Ne}$ trajectories which were taken from the same ${ }^{17} \mathrm{Ne}+\mathrm{p}_{1}+\mathrm{p}_{2}$ event. (a) Distribution for events with large $\mathrm{p}^{17} \mathrm{Ne}$ angles $(>50 \mathrm{mrad})$. (b) Distribution for events with small $\mathrm{p}-{ }^{17} \mathrm{Ne}$ angles $(<50 \mathrm{mrad})$.

measured with an accuracy of $0.1 \mathrm{~mm}$. Angular uncertainties of the decay products were mainly due to the angular straggling of protons in the MSD and amounted to $\sim 1 \mathrm{mrad}$.

Additional technical information concerning the detectors and their electronics as well as the applied tracking procedures can also be found in Refs. [23,26,27].

\section{NUCLEAR-STRUCTURE INFORMATION FROM PROTON-ION ANGULAR CORRELATIONS}

In the following section we want to show how nuclearstructure information concerning the nuclei involved in $1 \mathrm{p}$ or $2 p$ decay can be obtained from measuring only the trajectories of the decay products, without measuring their kinetic energies.

For a discussion of $2 p$ decay, we can discriminate between three different cases. These cases are illustrated in Fig. 10. The upper panels show schematically the nuclear states involved in $2 \mathrm{p}$ decay, the lower panels show the corresponding momentum correlations $k_{p 1-\mathrm{HI}}-k_{p 2-\mathrm{HI}}$. In Fig. 10(a) of direct $2 \mathrm{p}$ decay, sequential $2 \mathrm{p}$ emission is energetically forbidden since even the g.s. of the intermediate nucleus with mass number $A-1$ lies higher in energy than the g.s. of the $2 \mathrm{p}$ precursor with mass number $A$. The theory of direct $2 p$ emission tells us $[5,7,28,29]$ that the momenta of both emitted protons should
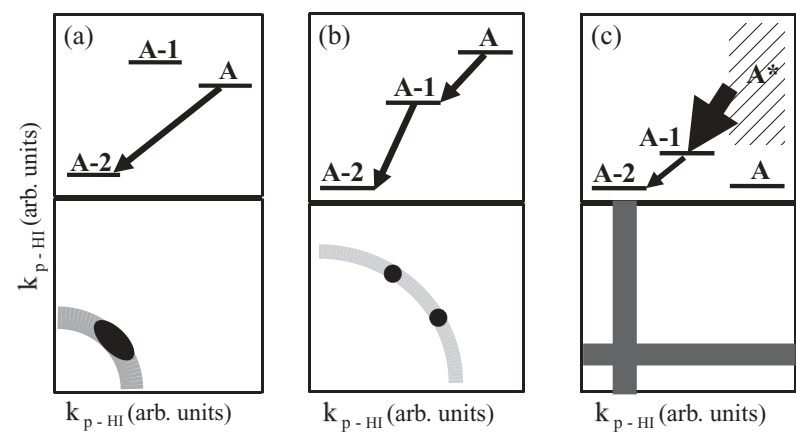

FIG. 10. Cartoons of transverse momentum correlations $k_{p 1-\mathrm{HI}}-$ $k_{p 2-\mathrm{HI}}$ (lower panels) expected for three different mechanisms of $2 \mathrm{p}$ emission (illustrated in the upper panels) from a parent nucleus with mass number $A$ to a daughter nucleus with $A$-2: (a) direct three-body decay, (b) sequential emission of protons via a narrow intermediate state in nucleus $A-1$, and (c) deexcitation of broad continuum states in the nucleus $A$ with final-state interaction due to a narrow resonance in the intermediate nucleus with $A-1$.

preferentially be equal; consequently, the $2 \mathrm{p}$ momentumcorrelation plot should have the shape of a quadrant, with a radius corresponding to the $Q_{2 p}$ value and with most of the counts lying in the peak indicated by the dark spot in the lower panel of Fig. 10(a). Note that all momentum-correlation plots in Fig. 10 are symmetric with respect to the $45^{\circ}$ line since the protons $p_{1}$ and $p_{2}$ are indistinguishable.

Figure 10(b) represents the sequential emission of two protons through a narrow resonance in the intermediate nucleus with $A-1$. The $k_{p 1-\mathrm{HI}}-k_{p 2-\mathrm{HI}}$ correlation plot should yield double peaks [30] as indicated by the black dots in the lower panel of Fig. 10(b).

The third $2 p$-decay mechanism to be considered is $2 p$ emission from broad continuum parent states with final-state interactions in the p-HI pairs [Fig. 10(c)]. This mechanism should reveal broad distributions along narrow "slices" as shown in the lower part of Fig. 10(c).

In the present method, we do not measure proton or HI momenta. Instead, we simply measure their trajectories directly downstream from the secondary-reaction target. Figure 11(a) shows the kinematics plot for the simple case of

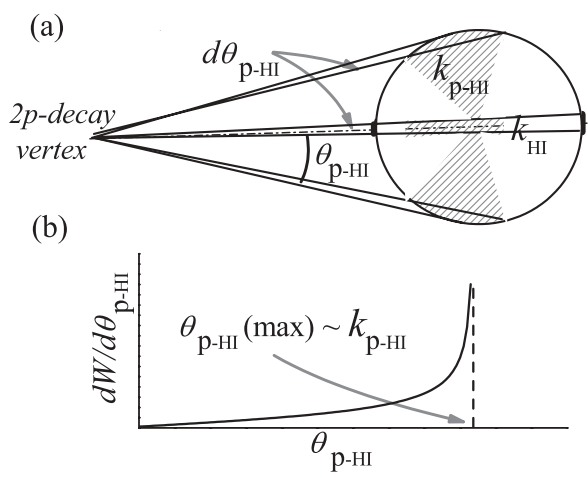

FIG. 11. (a) Kinematics of isotropic mono-energetic proton emission from a high-energy heavy ion. (b) The corresponding angular p-HI distribution exhibiting the peak corresponding to the $Q$-value of the proton decay. 
isotropic and mono-energetic single-proton emission from a high-energy heavy ion. Figure 11(b) shows the corresponding distribution of laboratory $\mathrm{p}-\mathrm{HI}$ opening angles, $\theta_{p-\mathrm{HI}}$. The angular spectrum exhibits a sharp peak corresponding to the situation where the proton is emitted roughly orthogonal to the HI momentum vector. Thus the maximum value of $\theta_{p-\mathrm{HI}}$ is directly related to the decay energy $Q_{p}$ of the emitted proton. In the same way, the $k_{p 1-\mathrm{HI}}-k_{p 2-\mathrm{HI}}$ momentum correlations for 2 p decays (Fig. 10) can be replaced by the corresponding $\theta_{p 1-\mathrm{HI}}-\theta_{p 2-\mathrm{HI}}$ correlations. If the initial and final states of $\mathrm{p}$ emission are narrow, the width of a peak in the angular distribution is governed mostly by angular straggling of the proton in the secondary-reaction target. If those states are broad, the width results from a convolution of the state's width with proton angular straggling.

It is clear that the cases sketched in Figs. 10 and 11 represent ideal cases. In reality, several proton branches may be present, representing more than one of the cases shown schematically in Figs. 10(a) through (c), and leading to complicated spectra with several peaks. One can, however, clean up the spectra and enhance transitions with small $Q$ values by gating on small angles of $\theta_{p 1-\mathrm{HI}}$ and plotting the spectrum of $\theta_{p 2-\mathrm{HI}}$ under this condition. Another option is to plot the one-dimensional distribution of $\rho=\sqrt{\theta_{p 1-\mathrm{HI}}^{2}+\theta_{p 2-\mathrm{HI}}^{2}}$ instead of the twodimensional distribution $\theta_{p 1-\mathrm{HI}}$ versus $\theta_{p 2-\mathrm{HI}}$. The variable $\rho$ is related to the energy sum of both emitted protons and therefore to the 2 p-decay energy $Q_{2 p}$ of the precursor state. The $\rho$ distributions are very useful in the analysis of $2 \mathrm{p}$ decay data since they produce spectra with less peaks and allow to gate on specific excitation-energy regions.

In all cases, detailed Monte Carlo simulations are needed to interpret the angular spectra. To obtain the energy of a resonance, its numerical value is varied within a reasonable range of energies and for each case the corresponding angular correlation is calculated. This predicted angular correlation is then compared to the measured one. The resonance energy is obtained as the test value where the probability that the two distributions are identical is maximum. In the same way, at least limits for the width of a resonance can be obtained, as will be shown in the following.

\section{INVESTIGATIONS OF REFERENCE CASES: 1P AND $2 P$ DECAYS OF KNOWN STATES IN ${ }^{15} \mathrm{~F},{ }^{16} \mathrm{NE}$, AND ${ }^{19} \mathrm{NA}$}

\section{A. $2 p$ decay of the ${ }^{16} \mathrm{Ne}$ ground state}

The validity of our method to obtain the properties of proton-unbound states from tracking their decay products can be tested by reproducing the literature data. For this purpose, we studied the $2 p$ decay of ${ }^{16} \mathrm{Ne}$ into ${ }^{14} \mathrm{O}+\mathrm{p}+\mathrm{p} .{ }^{16} \mathrm{Ne}$ was formed by $1 \mathrm{n}$-removal inside the reaction target from the ${ }^{17} \mathrm{Ne}$ secondary beam, which was transmitted simultaneously with the ${ }^{20} \mathrm{Mg}$ secondary beam. Its $2 \mathrm{p}$ decay can be gated on by requiring two coincident proton tracks in the microstrip detectors simultaneously with identifying ${ }^{14} \mathrm{O}$ in the second half of the FRS (see Fig. 3).

A scheme of the relevant levels in ${ }^{16} \mathrm{Ne},{ }^{15} \mathrm{~F}$, and ${ }^{14} \mathrm{O}$ is shown in Fig. 12. The 2 p-decay energy of the ${ }^{16} \mathrm{Ne}$ g.s. was measured with high precision to be $1.40(2) \mathrm{MeV}$ [31]. Its decay

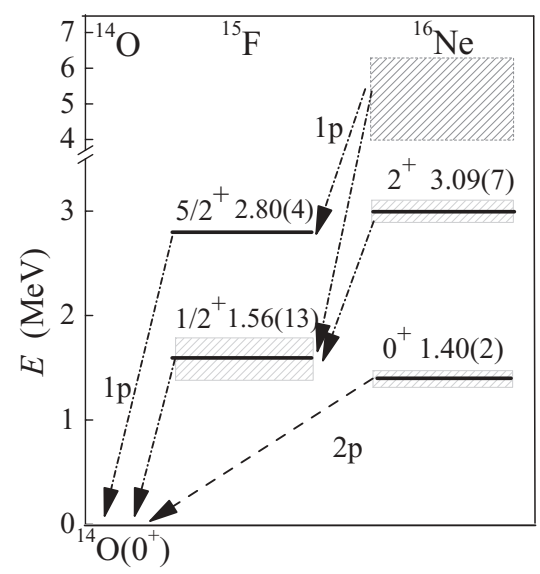

FIG. 12. The level schemes of known states in ${ }^{14} \mathrm{O},{ }^{15} \mathrm{~F}$, and ${ }^{16} \mathrm{Ne}$ (Refs. [12-14,31,32]). 1p decays of states in ${ }^{16} \mathrm{Ne}$ and ${ }^{15} \mathrm{~F}$ into the g.s. of ${ }^{14} \mathrm{O}$ are indicated by the dash-dotted arrows. The $2 \mathrm{p}$ decay of the ${ }^{16} \mathrm{Ne}$ g.s. is illustrated by the dashed arrow. The hatched area indicates the unspecified continuum states in ${ }^{16} \mathrm{Ne}$. Decay energies and level widths are given in $\mathrm{MeV}$; the decay energies are relative to the respective $1 \mathrm{p}$ and $2 \mathrm{p}$ thresholds.

properties and those of the related resonances in intermediate ${ }^{15} \mathrm{~F}$ were also studied in Refs. [12-14,32]. The $2 \mathrm{p}$ emission of the ${ }^{16} \mathrm{Ne}$ g.s. must be mainly direct decay because the sequential emission of protons via an intermediate resonance, the (higher lying) g.s. of ${ }^{15} \mathrm{~F}$, is energy forbidden and can only proceed through the tails of the respective resonances (see Fig. 12). The quantum-mechanical theory of direct $2 p$ decay which uses a three-body model [5-7] predicts the correlations of the fragments of ${ }^{16} \mathrm{Ne}$ to be strongly influenced by nuclear structure together with Coulomb and three-body centrifugal barriers. In particular, p-p correlations are expected to result from a dominant $s$-wave configuration in the ${ }^{16} \mathrm{Ne}$ g.s. (in contrast with its mirror, ${ }^{16} \mathrm{C}$, thus breaking isospin symmetry) [33].

\section{Monte Carlo simulations}

To understand the conversion from momentum to angular correlations in $2 p$ decay, we performed simulations of $2 p$ decays from the known g.s. of ${ }^{16} \mathrm{Ne}$ under the assumption that its mechanism is direct $2 \mathrm{p}$ emission. Monte Carlo simulations were done using the GEANT software package [34]. GEANT includes relativistic kinematics and all important processes of interaction of relativistic particles with various materials. The physical input for the GEANT simulations was provided by the three-body model mentioned previously [5-7]. The simulations started at a randomly chosen $\mathrm{Hi}+\mathrm{p}+\mathrm{p}$ vertex inside the reaction target and followed the protons and $\mathrm{HI}$ through the remainder of the reaction target and the detectors, taking into account angular straggling and detection efficiency as well as the experimental uncertainties in position determination and in reconstructing the vertex coordinates, trajectory angles, and so on. In particular, all selection conditions applied for a data analysis were used in simulations in the same way.

The result of the simulation for direct $2 p$ decay of the ${ }^{16} \mathrm{Ne}$ g.s. is shown in Fig. 13(a). Its similarity with the 

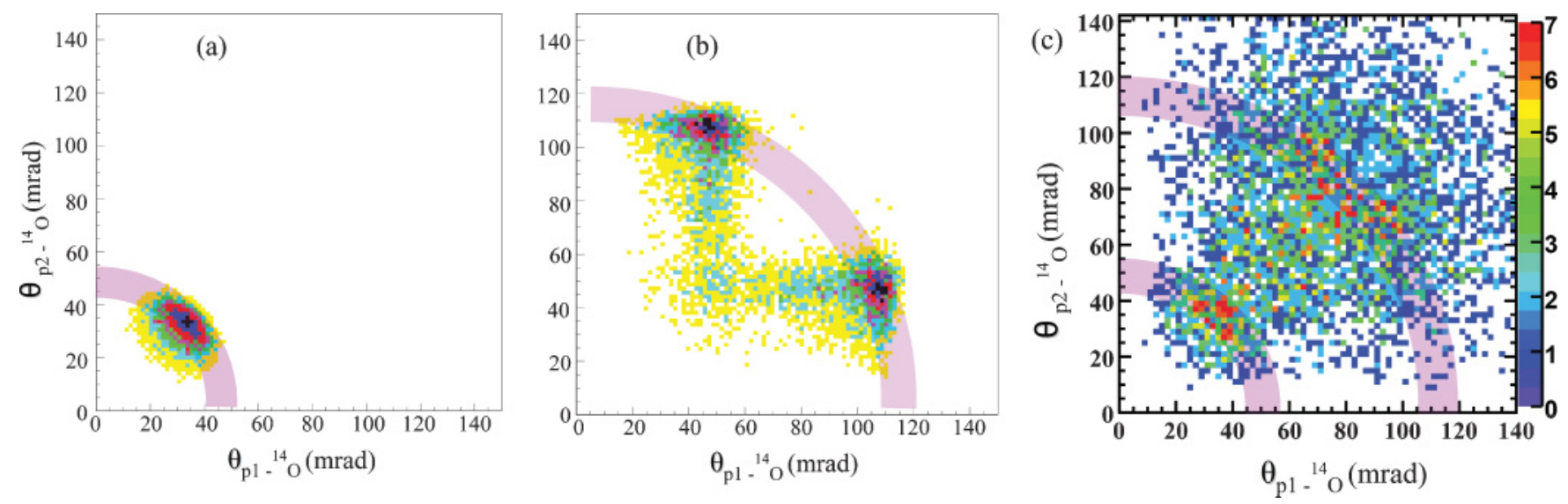

FIG. 13. (Color online) (a) Simulated angular correlations $\theta_{p 1 \_14}-\theta_{p 2 \_14}$ from Monte-Carlo simulations of the experimental response to the direct $2 \mathrm{p}$ decay of the ${ }^{16} \mathrm{Ne}$ g.s. with a $2 \mathrm{p}$-decay energy of $1.4 \mathrm{MeV}$. (b) Similar simulation for the $2 \mathrm{p}$ decay of an assumed excited state in ${ }^{16} \mathrm{Ne}$ with a $2 \mathrm{p}$-decay energy of $7.6 \mathrm{MeV}$ decaying sequentially by $2 \mathrm{p}$ emission through the g.s. of ${ }^{15} \mathrm{~F}$. (c) Experimental angular correlations $\left(\mathrm{p}_{1}-{ }^{14} \mathrm{O}\right)-\left(\mathrm{p}_{2}-{ }^{14} \mathrm{O}\right)$ obtained from measured ${ }^{14} \mathrm{O}+\mathrm{p}+\mathrm{p}$ events (color boxes with scale shown on the right-hand side). The shadowed arc areas indicate the locations of simultaneous or sequential $2 \mathrm{p}$ decays of the most intensively populated states in ${ }^{16} \mathrm{Ne}$ (the ground and the $7.6-\mathrm{MeV}$ excited state).

schematic plot in Fig. 10(a) is obvious. The $2 p$ decay energy $Q_{2 p}=1.40(2) \mathrm{MeV}$ [31] is translated into an arc with a radius of about $45 \mathrm{mrad}$; projecting the distribution leads to a single peak at about $35 \mathrm{mrad}$.

In a similar way, the sequential-decay mechanism [shown schematically in Fig. 10(b)] can be simulated. An example is displayed in Fig. 13(b). It represents the sequential decay of a hypothetical broad state in ${ }^{16} \mathrm{Ne}$ with a $2 \mathrm{p}$-decay energy of $7.6 \mathrm{MeV}$ via the g.s. of ${ }^{15} \mathrm{~F}$. According to its schematic representation in Fig. 10, it results in two symmetric bumps (at 50 and $110 \mathrm{mrad}$ in the projection onto one axis). The arc radius corresponds to about $115 \mathrm{mrad}$.

\section{Comparison with experimental data for ${ }^{14} O+p+p$}

Figure 13(c) shows the measured angular $\left(\mathrm{p}_{1^{-}}{ }^{14} \mathrm{O}\right)-\left(\mathrm{p}_{2}{ }^{14} \mathrm{O}\right)$ correlations obtained in our experiment. The distribution is symmetrized with respect to proton permutations, proton indexes are given for illustration purposes only. One can distinguish several peaks indicating the most intense $2 \mathrm{p}$-emission channels. Events with the smallest $\mathrm{p}-{ }^{14} \mathrm{O}$ angles fall into a distinct cluster around $\theta_{p-\mathrm{O}}=35 \mathrm{mrad}$; these events can be attributed to the direct $2 p$ decay from the ${ }^{16} \mathrm{Ne}$ g.s. Most of the other events are located in an arc with a radius of about $115 \mathrm{mrad}$. This group can be attributed to the $2 p$ decay from a high-lying excited state in ${ }^{16} \mathrm{Ne}$.

For a quantitative interpretation, projections of the two-dimensional $\left(\mathrm{p}_{1}{ }^{14} \mathrm{O}\right)-\left(\mathrm{p}_{2}-{ }^{14} \mathrm{O}\right)$ distribution shown in Fig. 13(c) are useful. However, an inclusive projection onto the one-dimensional $\mathrm{p}-{ }^{14} \mathrm{O}$ axis as shown in Fig. 14(a) leads to a complicated spectrum with several peaks and the most intense peaks are labeled (1-4). To disentangle the "ground state" from the "excited state" events, one needs to project onto one $\mathrm{p}-{ }^{14} \mathrm{O}$ axis by gating on either the small-angle or large-angle regions of the other $\mathrm{p}^{14} \mathrm{O}$ axis. Such projections are shown
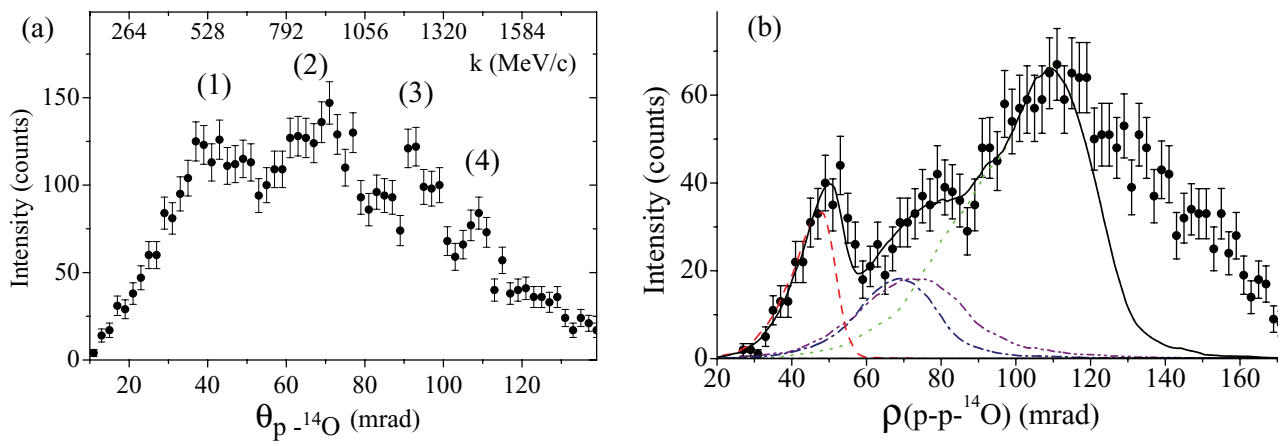

FIG. 14. (Color online) (a) Opening angles $\theta_{p-O}$ (full circles with statistical uncertainties) projected from the measured ${ }^{14} \mathrm{O}+\mathrm{p}+\mathrm{p}$ events shown in Fig. 13(c). The upper axis shows the transverse momenta $k$ of protons with respect to ${ }^{14} \mathrm{O}$. The apparent peaks are labeled (1-4). (b) Angular $\rho=\sqrt{\theta_{p 1-\mathrm{O}}^{2}+\theta_{p 2-\mathrm{O}}^{2}}$ distribution (full circles with statistical uncertainties) obtained from the data shown in Fig. 13(a). The dashed and dash-dotted curves show the results of Monte Carlo simulations of the experimental response to $2 \mathrm{p}$ decays of the known ground $0^{+}$and first-excited $2^{+}$states in ${ }^{16} \mathrm{Ne}$ with $Q_{2 p}$ values of 1.35 and $3.2 \mathrm{MeV}$, respectively. For illustration purpose, the dash-dot-dotted curve shows a hypothetical contribution from the excited second $0^{+}$state at $3.5 \mathrm{MeV}$. The dotted curve is the contribution of a previously unknown excited state with $Q_{2 p} \sim 7.6 \mathrm{MeV}$ (the properties of the excited states will be considered in Sec. V). The solid curve results from the sum fit. 

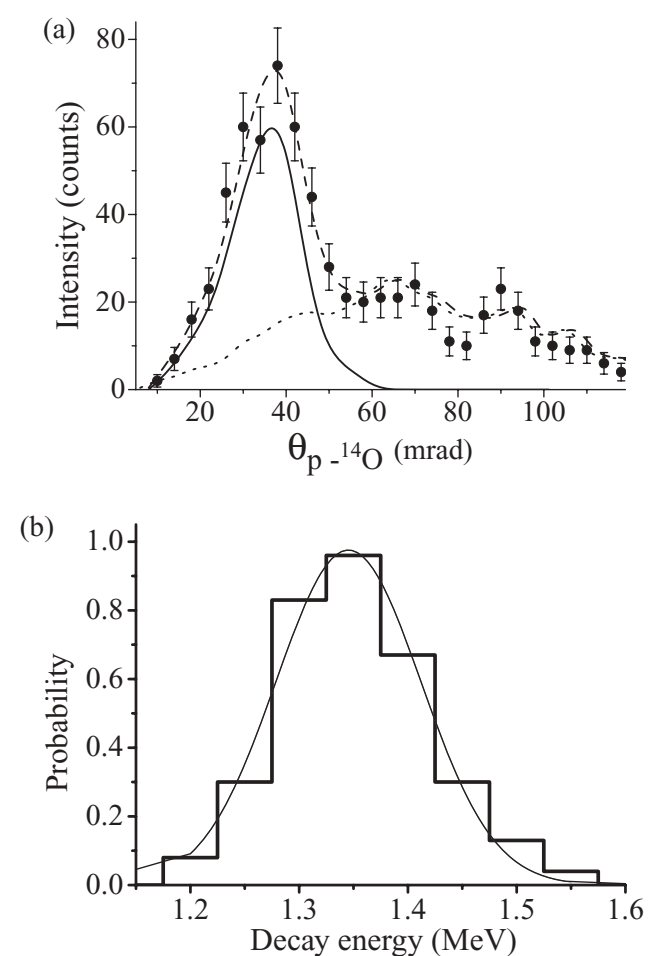

FIG. 15. (a) Angular $\mathrm{p}^{14} \mathrm{O}$ correlations (full circles with statistical uncertainties) obtained from the measured $\mathrm{p}+\mathrm{p}+{ }^{14} \mathrm{O}$ events by selecting the other proton angle $\theta_{p 2-O}$ within the range from 0 to $45 \mathrm{mrad}$, which corresponds to the ${ }^{16} \mathrm{Ne}$ g.s. region. The solid curve represents the Monte Carlo simulation of the detector response for ${ }^{16} \mathrm{Ne}_{\text {g.s. }} \rightarrow{ }^{14} \mathrm{O}+\mathrm{p}+\mathrm{p}$ with the fitted 2p-decay energy of $1.35(8) \mathrm{MeV}$. The dashed line is a sum fit to the data. The dotted curve indicates the assumed background contribution. (b) Calculated probability that the simulation matches the data as a function of the assumed decay energy (see text). The curve represents a Gaussian function fitted to the histogram.

in Figs. 15 and 17 and will be discussed in detail in the following. Another helpful presentation of the measured triple ${ }^{14} \mathrm{O}+\mathrm{p}+\mathrm{p}$ events is the distribution of $\rho=\sqrt{\theta_{p 1-\mathrm{O}}^{2}+\theta_{p 2-\mathrm{O}}^{2}}$ distribution as discussed at the end of Sec. III. We present this distribution in Fig. 14(b). As expected, the number of peaks was reduced and the spectrum exhibits more structure. One can clearly distinguish between the small-angle peak at $\sim 45 \mathrm{mrad}$ and a broad distribution at larger angles. Our simulations show that the small-angle peak is due to the $2 p$ decay of the ${ }^{16} \mathrm{Ne}$ g.s., and the continuous component is related to $2 p$ decays of excited states in ${ }^{16} \mathrm{Ne}$ (these states will be considered in Sec. V).

The upper panel in Fig. 15 displays the angular distribution $\theta_{p 1-0}$. The $\theta_{p 2-\mathrm{O}}$ gate was chosen from 0 to $45 \mathrm{mrad}$, where the $2 \mathrm{p}$ decay of the ${ }^{16} \mathrm{Ne}$ g.s. is expected to show up. The peak around $35 \mathrm{mrad}$ (the suggested "ground state") dominates the spectrum; only a few correlations are seen between a proton from the "ground state" and another proton at larger angles. This means that the protons from the "ground-state" and "excited-state" peaks are not correlated (i.e., the peaks cannot be explained by a sequential emission of protons from the same state in ${ }^{16} \mathrm{Ne}$ ). The large-angle part of the distribution (corre- sponding to larger excitation energies in ${ }^{16} \mathrm{Ne}$ ) has the same shape as the $\theta_{p 1-O}$ distribution selected within the $\theta_{p 2-O}$ range just outside the "ground state" region, from 48 to $160 \mathrm{mrad}$. By scaling this component to the large-angle data of the upper part of Fig. 15 we obtain the dotted background curve.

To interpret this spectrum quantitatively, the data are compared to a Monte Carlo simulation of the response of our setup to the $2 p$ decay ${ }^{16} \mathrm{Ne} \rightarrow{ }^{14} \mathrm{O}+\mathrm{p}+\mathrm{p}$ as described in Sec. IV A1. As inputs into the GEANT simulations, we used the predictions of fragment correlations in the precursor frame as given by the model of direct $2 p$ emission [33]. For a chosen range of $2 \mathrm{p}$-decay energies $Q_{2 p}$, we calculated the respective probabilities $P\left(Q_{2 p}\right)$ for the simulations to match the data (the standard statistical Kolmogorov-Smirnov test of compatibility of two histograms, see, e.g., [35]), which are shown in the lower panel in Fig. 15. The $Q_{2 p}$ value with the closest match (assuming that $P>50 \%$ ) was accepted. The best-fit simulation reproduces the data in the small-angle peak region quantitatively with $Q_{2 p}=1.35(8) \mathrm{MeV}$, in agreement with the literature value of $1.40(2) \mathrm{MeV}$ [31]. The statistical uncertainty of the fitted value is given by the $Q_{2 p}$ range where the experimental data are described by the simulation with probabilities above $50 \%$. One should note that the ungated calculations have practically the same shape as those cut by horizontal slices and therefore the corresponding systematic error may be neglected.

Figure 16 shows the angular $\mathrm{p}^{-14} \mathrm{O}$ correlations from the ${ }^{16} \mathrm{Ne}$ ground-state $2 \mathrm{p}$ decay selected by another gate, namely the arc-area covering the g.s. decay correlations as shown in Fig. 13(c). Such a gate cuts off most of the continuous background, but changes the shape of the distribution a bit as demonstrated by the gated and ungated calculations of the corresponding correlations. As the applied selection gate on the $\rho$ parameter may introduce some uncontrolled bias in the generated distribution in comparison with the previous case, we accept the fit results illustrated in Fig. 15 because their systematic errors are under control.

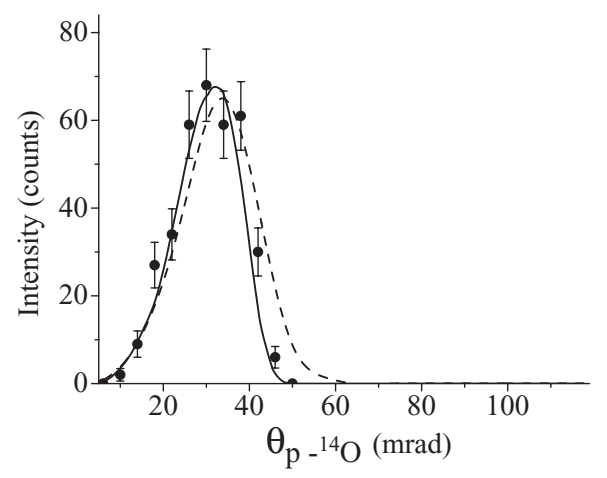

FIG. 16. Angular $\mathrm{p}^{-14} \mathrm{O}$ correlations (full circles with statistical uncertainties) from the ${ }^{16} \mathrm{Ne}$ g.s. obtained from the measured $\mathrm{p}+\mathrm{p}+{ }^{14} \mathrm{O}$ events by selecting their arc-area $\rho=\sqrt{\theta_{p 1-\mathrm{O}}^{2}+\theta_{p 2-\mathrm{O}}^{2}}$ within the range from 35 to $50 \mathrm{mrad}$ [see Fig. 14(b)]. The solid curve is the corresponding calculation of the decay ${ }^{16} \mathrm{Ne}_{\mathrm{g} . \mathrm{s}} \rightarrow{ }^{14} \mathrm{O}+\mathrm{p}+\mathrm{p}$ with a 2p-decay energy of $1.35(8) \mathrm{MeV}$ cut by the same condition as the data. The similar but un-truncated calculation is presented by the dashed curve whose shape practically coincides with the calculation in Fig. 15(a). 


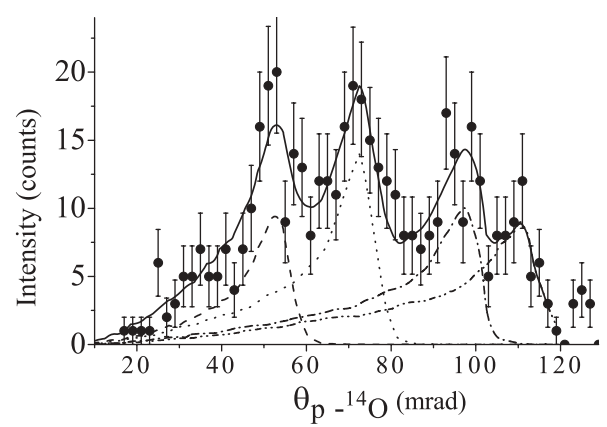

FIG. 17. Angular $\mathrm{p}^{14} \mathrm{O}$ distribution obtained from the data shown in Fig. 13(c) by selecting the other proton angle from 120 to $150 \mathrm{mrad}$, which corresponds to $\mathrm{p}-{ }^{14} \mathrm{O}$ final-state interactions due to the ground and excited states of ${ }^{15} \mathrm{~F}$. The dashed and dotted curves result from Monte Carlo simulations of the known $1 \mathrm{p}$ decays of the ground and first-excited states of ${ }^{15} \mathrm{~F}$ with $1 \mathrm{p}$-decay energies of $1.56(13)$ and 2.80(4) MeV, respectively [12]. The dash-dotted and dash-dot-dotted curves show the fit of two other peaks by suggesting two unknown excited states in ${ }^{15} \mathrm{~F}$ with 1p-decay energies of $4.9(2)$ and $6.4(2) \mathrm{MeV}$, respectively. Their derived properties are given in Sec. V. The solid line is the sum fit.

\section{B. 1p decay of known states in ${ }^{15} \mathrm{~F}$}

The measured $p+p+$ HI events can also be used for studies of 1p-decaying resonances in unbound nuclei. Figure 17 displays the angular $\mathrm{p}-{ }^{14} \mathrm{O}$ distribution for the continuum of highly "excited states" in ${ }^{16} \mathrm{Ne}$ [see Fig. 13(c)] obtained from the $\mathrm{p}+\mathrm{p}+{ }^{14} \mathrm{O}$ events by selecting large angles of the other proton ranging from 120 to $160 \mathrm{mrad}$. The selected range corresponds to energies of the other proton between $\sim 8$ and $\sim 20 \mathrm{MeV}$. The distribution shows two small-angle peaks that reflect the $\mathrm{p}-{ }^{14} \mathrm{O}$ final-state interaction due to the ground and first-excited states in ${ }^{15} \mathrm{~F}$. This can be verified by Monte Carlo simulations of $1 \mathrm{p}$ decays ${ }^{15} \mathrm{~F}^{*} \rightarrow{ }^{14} \mathrm{O}+\mathrm{p}$ of the known ground $1 / 2^{+}$and first-excited $5 / 2^{+}$states in ${ }^{15} \mathrm{~F}$ with $Q_{p}$ values of 1.56(13) and 2.80(4) MeV [12], respectively. With these assumptions, the first two small-angle peaks are reproduced quantitatively. The peaks at larger angles are assigned to $1 \mathrm{p}$ decays of previously unknown excited states in ${ }^{15} \mathrm{~F}$ and the respective spectroscopic analysis is described in Sec. V.

\section{1p decay of the ${ }^{19} \mathrm{Na}$ ground state}

Another reference case is the $1 \mathrm{p}$ decay of the known g.s. of ${ }^{19} \mathrm{Na}$ whose decay energy is $Q_{p}=0.32(2) \mathrm{MeV}$ [36]. It is fed by the decay of high-lying continuum states in ${ }^{20} \mathrm{Mg}$, as shown schematically in Fig. 10(c). We detected it in the measured ${ }^{18} \mathrm{Ne}+\mathrm{p}+\mathrm{p}$ coincidence events whose angular $\left(\mathrm{p}_{1}{ }^{18} \mathrm{Ne}\right)-\left(\mathrm{p}_{2}{ }^{-18} \mathrm{Ne}\right)$ correlations are shown in Fig. 18(a). For illustration purposes, the corresponding Monte Carlo simulations are shown in Fig. 18(b).

Projecting the two-dimensional angular distribution shown in Fig. 18(a) onto the $\theta_{\mathrm{p}-18} \mathrm{Ne}$ axis yields a one-dimensional spectrum the lower part of which is shown in Fig. 19(a). A narrow peak around $25 \mathrm{mrad}$ corresponds to the expected $1 \mathrm{p}$ decaying resonance, the g.s. of ${ }^{19} \mathrm{Na}$. The assumed background is extrapolated from the data outside the ${ }^{19} \mathrm{Na}$ peak and contributes by $\sim 27 \%$ in the peak region.
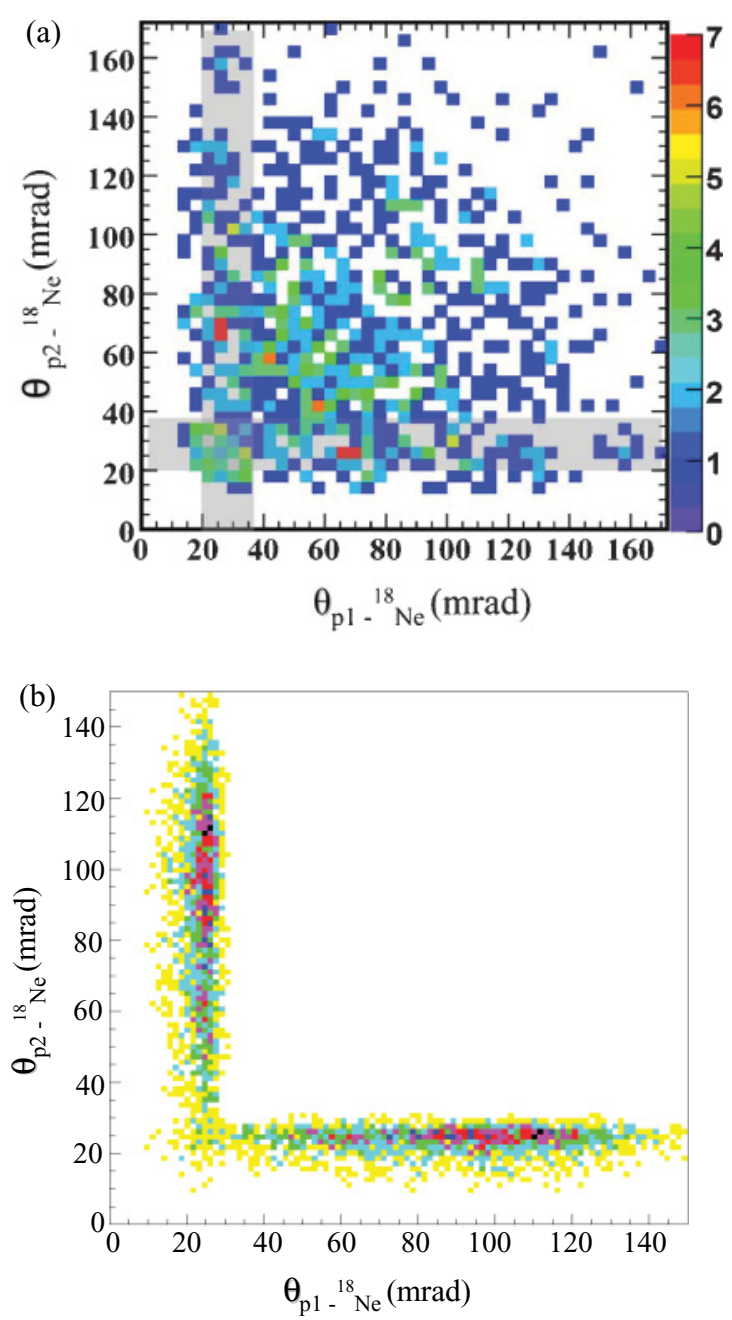

FIG. 18. (Color online) (a) Angular $\left(\mathrm{p}_{1}{ }^{-18} \mathrm{Ne}\right)-\left(\mathrm{p}_{2}{ }^{-18} \mathrm{Ne}\right)$ correlations obtained from the measured ${ }^{18} \mathrm{Ne}+\mathrm{p}+\mathrm{p}$ events (color boxes with scale shown on the right-hand side). The grey slices indicate the areas where final-state interaction in the $\mathrm{p}-{ }^{18} \mathrm{Ne}$ pairs due to the ${ }^{18} \mathrm{Na}$ g.s. is expected. (b) Angular correlations $\theta_{p 1 \_18 \mathrm{Ne}}-\theta_{p 2{ }^{18} \mathrm{Ne}}$ obtained by Monte Carlo simulations of the experimental response for $2 p$ emission from broad continuum states in ${ }^{20} \mathrm{Mg}$ through the g.s. of ${ }^{19} \mathrm{Na}$ with a known 1p-decay energy of 0.32 (2) $\mathrm{MeV}$ [36].

Monte Carlo simulations of the detector response for the decay of ${ }^{19} \mathrm{Na}_{\text {g.s. }} \rightarrow{ }^{18} \mathrm{Ne}+\mathrm{p}$ were performed to obtain the bestfit value for the $Q_{p}$ value of this decay. Figure 19(b) shows the probabilities that the simulations match the data as a function of the assumed $1 \mathrm{p}$-decay energy. The highest probability is obtained for $Q_{p}=0.328(22) \mathrm{MeV}$, which is in quantitative agreement with the literature value of 0.321 (13) $\mathrm{MeV}$ [36]. Using the same method, we have also tried to determine the width of the ${ }^{19} \mathrm{Na}$ g.s. Varying the width yields the probability distribution shown in Fig. 19(c). This gives only an upper limit of less than $40 \mathrm{keV}$. As the width of the ${ }^{19} \mathrm{Na}_{\text {g.s. }}$ is known to be very small, $<1 \mathrm{eV}$, our result reflects the experimental resolution of our detector setup.

At the end of this section we emphasize that all our nuclearstate assignments assume that one peak in the measured p-HI spectra matches one single resonance only. Therefore several 

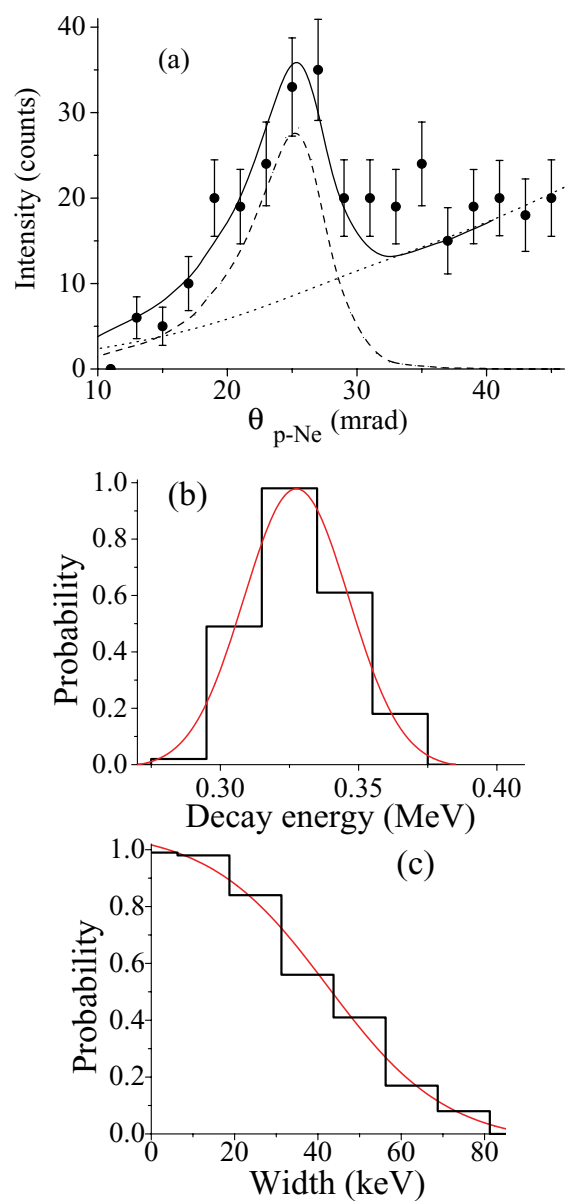

FIG. 19. (Color online) (a) Angular p- ${ }^{18} \mathrm{Ne}$ correlations (full circles with statistical uncertainties) projected from Fig. 18(a). The peak reflects the final-state interaction due to the ${ }^{19} \mathrm{Na}$ g.s. The dashed curve represents the Monte Carlo simulation of the detector response for ${ }^{19} \mathrm{Na}_{\text {g.s. }} \rightarrow{ }^{18} \mathrm{Ne}+\mathrm{p}$ with the fitted $1 \mathrm{p}$-decay energy of $0.328(22) \mathrm{MeV}$. The assumed background (dotted line) is interpolated from the data outside the ${ }^{19} \mathrm{Na}$ peak. The solid line is a sum fit to the data. The lower panels show the probability that the simulations match the data as functions of (b) the assumed decay energy and (c) width of the ${ }^{19} \mathrm{Na}$ g.s. The probability histograms are fitted by Gaussian and sigmoidal functions (solid lines).

closely spaced states (e.g., in the case of $1 \mathrm{p}$ decays of the lowest states in ${ }^{16} \mathrm{~F}$ ) can be misinterpreted as one broad level if they are populated within the experimental resolution of $0.2-0.4 \mathrm{MeV}$. Simulations of the response of our setup show that multiple scattering of the protons in the thick target is the main reason for the p-HI peak broadening and the relatively large errors of the resonance-width measurements. The obtained spectroscopic information may be improved in new experiments by using a thinner reaction target.

\section{SPECTROSCOPY OF HITHERTO UNKNOWN 1P- AND 2P-UNBOUND STATES IN ${ }^{15} \mathrm{~F}$ AND ${ }^{16} \mathrm{NE}$}

In the previous section, we demonstrated that we can precisely reproduce the known properties of proton-decaying resonances with our tracking technique. This has encouraged us to deduce the properties of so far unknown states in ${ }^{15} \mathrm{~F}$ and ${ }^{16} \mathrm{Ne}$ with the same method.

\section{A. States in ${ }^{15} \mathbf{F}$}

As mentioned in Secs. IV A2 and IV B, projections of the two-dimensional distribution of $\left(\mathrm{p}_{1}{ }^{-14} \mathrm{O}\right)-\left(\mathrm{p}_{2}{ }^{-}{ }^{14} \mathrm{O}\right)$ correlations [Fig. 13(c)] from the decay of ${ }^{16} \mathrm{Ne}$ exhibit peaks that can either be attributed to the direct $2 p$ decay of the ${ }^{16} \mathrm{Ne}$ g.s. or to the sequential decay of excited states in ${ }^{16} \mathrm{Ne}$ via two known states in ${ }^{15} \mathrm{~F}$, the $1 / 2^{+}$g.s. or $5 / 2^{+}$first-excited state. These events were disentangled by making slice projections from the measured $\left(\mathrm{p}_{1}{ }^{-14} \mathrm{O}\right)-\left(\mathrm{p}_{2}-{ }^{14} \mathrm{O}\right)$ correlations, selecting a small-angle gate, $\theta_{p 2-}<45 \mathrm{mrad}$ to enhance the direct $2 \mathrm{p}$ g.s. decay, and a large-angle gate, $\theta_{p 2-O}>120 \mathrm{mrad}$ to enhance the sequential decays.

Figure 20(b) again displays the $\theta_{p 1-0}$ distribution obtained by gating on condition $\theta_{p 2-0}>120 \mathrm{mrad}$, as in Fig. 17. For illustration purposes, the inclusive $\theta_{p 1-\mathrm{O}}$ distribution is shown in Fig. 20(a). The two left-most peaks in Fig. 20(b) were attributed in Sec. IV B to $1 \mathrm{p}$ decays of the known ground $1 / 2^{+}$and first-excited $5 / 2^{+}$states in ${ }^{15} \mathrm{~F}$ with $Q_{p}$ of $1.5(1)$ and 2.8(1) $\mathrm{MeV}$, respectively. The two peaks at larger angles can be assigned to $1 \mathrm{p}$ decays of unknown excited states in ${ }^{15} \mathrm{~F}$ with derived $Q_{p}$ values of 4.9(2) and 6.4(2) MeV [21]. These values and their uncertainties were deduced in the same way as those derived for ${ }^{16} \mathrm{Ne}$, by varying their level energies in the Monte-Carlo simulation of sequential decays until the simulated spectra matched the measured ones. In a similar way, the resonance widths $\Gamma$ were fitted, the $1 / 2^{+}$and $5 / 2^{+}$states in ${ }^{15} \mathrm{~F}$ with known widths serving as test cases. The levels with $Q_{p}$ of 4.9 and $6.4 \mathrm{MeV}$ are very narrow and smaller than our experimental resolution, the upper limit amounts to $0.2 \mathrm{MeV}$. In the inset of Fig. 20(b) we plot the probability distribution, $P(\Gamma)$, for the width $\Gamma$ of the level with $Q_{p}=4.9 \mathrm{MeV}$.

The state with $Q_{p}=4.9 \mathrm{MeV}$ is likely the mirror state of ${ }^{15} \mathrm{C}\left(\frac{1}{2}^{-}\right)$since its location in ${ }^{15} \mathrm{~F}$ relative to the $\left(\frac{5}{2}^{+}\right)_{1}$ state is similar to that in ${ }^{15} \mathrm{C}$. This state is $0.27 \mathrm{MeV}$ above the $2 \mathrm{p}$ threshold, however, the ${ }^{14} \mathrm{O}\left(0_{1}^{+}\right)+\mathrm{p} 1 \mathrm{p}$-decay mode dominates.

The $Q_{p}=6.4 \mathrm{MeV}$ state in ${ }^{15} \mathrm{~F}$, observed in the ${ }^{14} \mathrm{O}\left(0_{1}^{+}\right)+\mathrm{p}$ decay, is also open for $2 \mathrm{p}$ decay via the $1_{1}^{-}, 0_{2}^{+}$, and $3_{1}^{-}$states in ${ }^{14} \mathrm{O}$ and may be seen in triple ${ }^{13} \mathrm{~N}+\mathrm{p}+\mathrm{p}$ coincidences. Their angular $\theta_{p_{1}-\mathrm{N}}-\theta_{p_{2}-\mathrm{N}}$ correlations are shown in Fig. 21(a). Only few events are detected in the arc area of interest around $62 \mathrm{mrad}$, so we may conclude that the $2 \mathrm{p}$-decay branch of the $Q_{p}=6.4 \mathrm{MeV}$ state has not been observed. Theoretical estimates of the widths $\Gamma$ of these unobserved decays can be obtained by the standard expression

$$
\Gamma=C^{2} S \cdot \Gamma_{\text {s.p. }} .
$$

Single-particle widths $\Gamma_{\text {s.p. }}$ were calculated in the two-body potential model with Woods-Saxon potential parameters of $r_{0}=1.25 \mathrm{fm}$ and $a=0.65 \mathrm{fm}$ (see, e.g., Ref. [14]). The spectroscopic factors $C^{2} S$ were determined from occupancies of bound orbits calculated in the ( $s p s d p f)$-shell model with the Warburton and Brown (WBP) interaction [37] using the NUSHELL@MSU code [38]. Two possible $J^{\pi}$ assignments for 

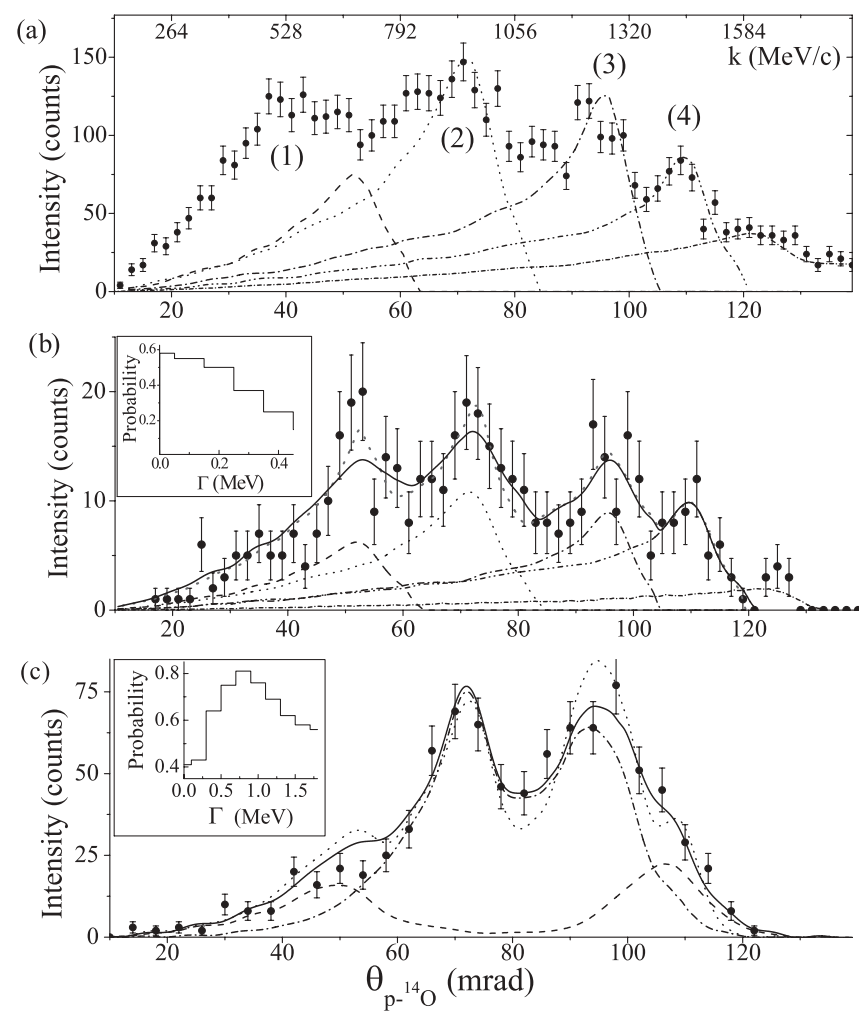

FIG. 20. (a) The $\theta_{p-O}$ correlations shown in Fig. 14(b) in comparison with the simulations of different $1 \mathrm{p}$ decays of ${ }^{15} \mathrm{~F}$ states. The simulation curves are similar to those described in (b). (b) Onedimensional $\theta_{p-o}$ projection (full circles with statistical uncertainties) of the two-dimensional data shown in Fig. 13(c), obtained by gating on $\theta_{p_{2}-\mathrm{O}}>120 \mathrm{mrad}$, which corresponds to ${ }^{15} \mathrm{~F}$ resonances due to final-state interactions in $p_{1}-{ }^{14} \mathrm{O}$ pairs. The dashed and dotted curves result from simulations of the response of our setup to the known $1 \mathrm{p}$ decays of the ground and first-excited states in ${ }^{15} \mathrm{~F}$, see Sec. IV B. The dash-dotted and dash-dot-dotted curves indicate two new states in ${ }^{15} \mathrm{~F}$ with fitted $Q_{p}$ values of $4.9(2)$ and $6.4(2) \mathrm{MeV}$, respectively. The solid line is the sum fit. The short-dash curved shows the sum fit with all level widths set to $1 \mathrm{keV}$, which illustrates the experimental resolution. The short-dash-dotted curve is the $1 \mathrm{p}$-decay estimate of the $7.8 \mathrm{MeV}$ state in ${ }^{15} \mathrm{~F}$. (c) The $\theta_{p-\mathrm{O}}$ distribution selected within the arc-area $\sqrt{\theta_{p 1-\mathrm{O}}^{2}+\theta_{p 2-\mathrm{O}}^{2}}$ around $115 \mathrm{mrad}$, which corresponds to the $7.6 \mathrm{MeV}$ state in ${ }^{16} \mathrm{Ne}$. The solid curve is a fit obtained by simulating the sequential $2 \mathrm{p}$ decay of the ${ }^{16} \mathrm{Ne}^{*}$ state via the g.s. (dashed curve) and the first-excited state (dash-dotted curve) in ${ }^{15} \mathrm{~F}$. The dotted curve shows a similar fit with the ${ }^{16} \mathrm{Ne}^{*}$ width set to $1 \mathrm{keV}$. The insets in (b) and (c) show the probability (as a function of the assumed resonance width) that the simulations match the data.

the $Q_{p}=6.4 \mathrm{MeV}$ state were considered, $5 / 2^{-}$and $3 / 2^{-}$. In both cases, the dominant structure is a $d$-wave proton coupled to the ${ }^{14} \mathrm{O}\left(1^{-}\right)$core. However, due to the centrifugal barrier, the $\Gamma_{\text {s.p. }}$ for this configuration is only $\sim 11 \mathrm{keV}$ while the same proton is well above the barrier for the ${ }^{14} \mathrm{O}\left(0_{1}^{+}\right)+\mathrm{p}$ branch with $\ell=1$. Although $C^{2} S$ for the $\ell=1$ branch is small, the ${ }^{14} \mathrm{O}\left(0_{1}^{+}\right)+\mathrm{p}$ width dominates being $\sim 50 \mathrm{keV}$ for $5 / 2^{-}$and more than $100 \mathrm{keV}$ for $3 / 2^{-}$. The corresponding widths for the ${ }^{14} \mathrm{O}\left(1^{-}\right)+$p decay are about 4 and $7 \mathrm{keV}$. This explains our nonobservation of the $2 \mathrm{p}$ decay of the $Q_{p}=6.4 \mathrm{MeV}$ state. Although both $J^{\pi}$ assignments predict relatively narrow widths consistent with the data, the energy split between the $Q_{p}=4.9$ and $6.4 \mathrm{MeV}$ states favors the $3 / 2^{-}$assignment since the same split between the $1 / 2^{-}$and $3 / 2^{-}$states is observed in the mirror nucleus ${ }^{15} \mathrm{C}$. The $3 / 2^{-}$assignment also agrees with the shell-model predictions [17] (see Table I).

Triple ${ }^{13} \mathrm{~N}+\mathrm{p}+\mathrm{p}$ coincidences were measured in the same experiment and provided evidence for the presence of a new state in ${ }^{15} \mathrm{~F}$. Both the two-dimensional data and different projections are shown in Figs. 21(a) through (d). Two intense peaks are seen around 50 and $65 \mathrm{mrad}$ in the inclusive projection, $\theta_{p-\mathrm{N}}$ [Fig. 21(b)]. We selected these peaks by imposing the condition $78 \mathrm{mrad} \leqslant \sqrt{\theta_{p 1-\mathrm{N}}^{2}+\theta_{p 2-\mathrm{N}}^{2}} \leqslant 88 \mathrm{mrad}$ [Fig. 21(c)]. In the corresponding $\theta_{p-\mathrm{N}}$ distribution, two distinguished peaks have positions and widths that match those from the sequential $2 \mathrm{p}$ decay of a narrow ${ }^{15} \mathrm{~F}^{*}$ resonance via the known level ${ }^{14} \mathrm{O}\left(2_{1}^{+}\right)$at $6.59 \mathrm{MeV}$ [39]. This is justified by the final-state-interaction channel ${ }^{13} \mathrm{~N}+\mathrm{p} \rightarrow{ }^{14} \mathrm{O}^{*}$ whose $\theta_{p-\mathrm{N}}$ correlations are shown in Fig. 21(d). The simulations of the known 5.17 and $6.59 \mathrm{MeV}$ states in ${ }^{14} \mathrm{O}$ [39] match the two most intense peaks of this distribution providing references for this case. The fitted $Q_{2 p}$ value for the ${ }^{15} \mathrm{~F}^{*}$ state is $3.2(2) \mathrm{MeV}$; its width is estimated to be $\Gamma=0.4(4) \mathrm{MeV}$, see the inset of Fig. 21(c). As a cross check, we simulated an alternative sequential $2 \mathrm{p}$ decay via the neighboring ${ }^{14} \mathrm{O}\left(3^{-}\right)$ state at $6.27 \mathrm{MeV}$ (the $2 \mathrm{p}$-decay energy of the parent state should then be $8.0 \mathrm{MeV}$ ), which fails to fit the data. The new ${ }^{15} \mathrm{~F}^{*}$ state is also open to the ${ }^{14} \mathrm{O}\left(0_{1}^{+}\right)+\mathrm{p}$ decay branch with $Q_{p}=7.8(2) \mathrm{MeV}$. We simulated this channel using the ${ }^{15} \mathrm{~F}^{*}$ energy and width derived from the observed $2 \mathrm{p}$ branch, see Fig. 20(b). Some data events may be attributed to the 1p decay, though contributions from other possible higher-lying states in ${ }^{15} \mathrm{~F}$ are unknown. Thus we estimate the ratio of the $1 \mathrm{p} / 2 \mathrm{p}$ decay branches of the $7.8 \mathrm{MeV}$ state to be less than 0.2 .

Three $J^{\pi}$ assignments were considered for this state, $\left(\frac{3}{2}^{+}\right)_{2}$, $\left(\frac{1}{2}^{-}\right)_{2}$, and $\left(\frac{5}{2}^{+}\right)_{2}$, based on known spins and parities in the corresponding range of excitation energies in the mirror nucleus ${ }^{15} \mathrm{C}$. The spsdpf shell-model widths of the main decay channels for each of these assignments are given in Table I. The $\left(\frac{1}{2}^{-}\right)_{2}$ assignment is clearly wrong. We cannot discriminate between the $\left(\frac{3}{2}^{+}\right)_{2}$ and $\left(\frac{5}{2}^{+}\right)_{2}$ assignments by using the measured $1 \mathrm{p} / 2 \mathrm{p}$ branching ratio because the $1 \mathrm{p}$ decay width cannot be reliably determined in our theoretical approach. However, this energy matches well the predictions made for $\left(\frac{5}{2}^{+}\right)_{2}$ in the multichannel algebraic scattering (MCAS) approach [16]. If this assignment is confirmed in the future, this will mean that we observed a large Thomas-Ehrman shift of $0.6 \mathrm{MeV}$ in an $s$-wave configuration built on an excited core.

\section{B. States in ${ }^{16} \mathrm{Ne}$}

In the search for reaction channels feeding the observed ${ }^{15} \mathrm{~F}$ states, we inspected the two peaks in Fig. 20(a) around the $\mathrm{p}-{ }^{14} \mathrm{O}$ angles of 70 and $100 \mathrm{mrad}$. These peaks were assumed to originate from sequential $2 \mathrm{p}$ decay of a single excited state in ${ }^{16} \mathrm{Ne}$ via ${ }^{15} \mathrm{~F}$. The $\theta_{p-\mathrm{O}}$ distribution, selected within the corresponding arc area $110 \mathrm{mrad}<\sqrt{\theta_{p 1-\mathrm{O}}^{2}+\theta_{p 2-\mathrm{O}}^{2}}<$ $125 \mathrm{mrad}$ [see Fig. 20(c)] can be explained by sequential 
(a)
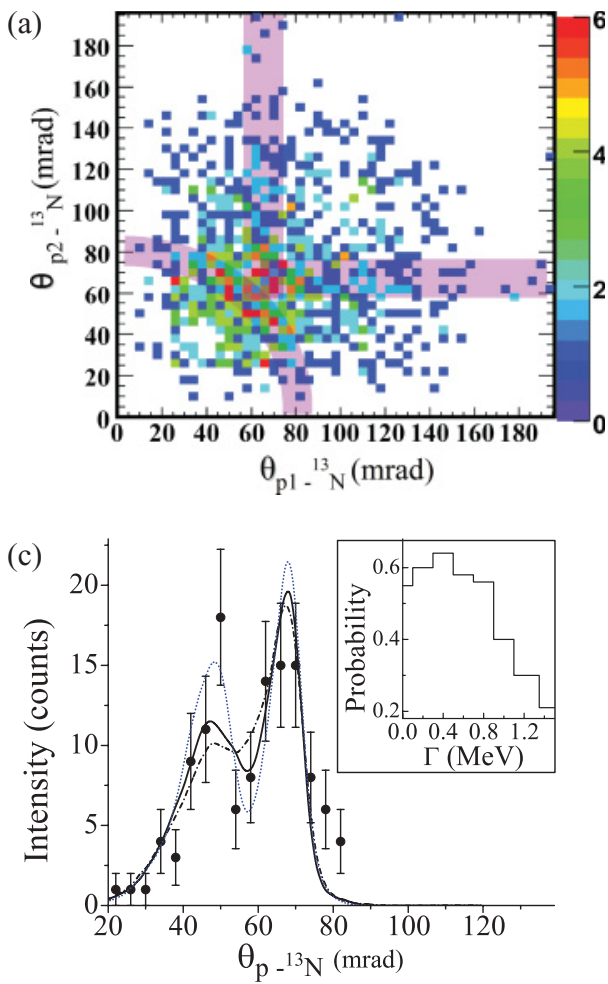

(b)

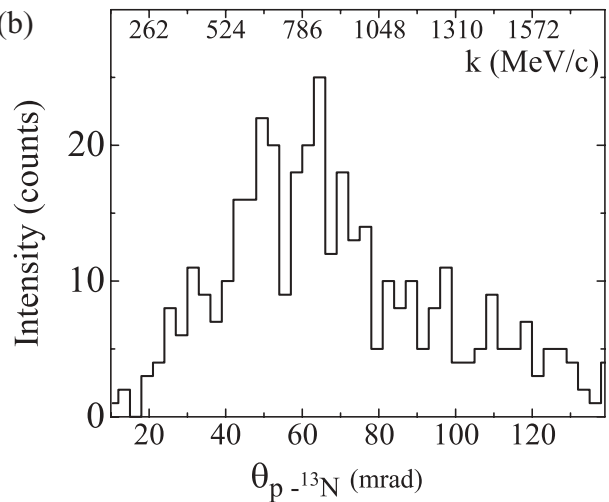

(d)

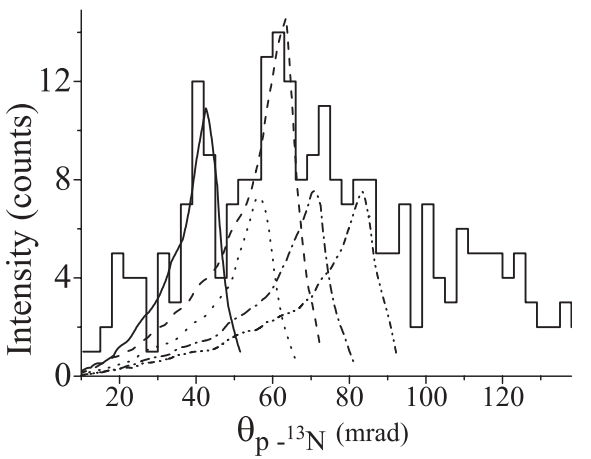

FIG. 21. (Color online) (a) Angular correlations $\theta_{p 1-\mathrm{N}}-\theta_{p 2-\mathrm{N}}$ from the ${ }^{13} \mathrm{~N}+\mathrm{p}+\mathrm{p}$ events (color boxes with scale shown on right). The arc area indicates $2 \mathrm{p}$ emission from an unknown ${ }^{15} \mathrm{~F}^{*}$ resonance. The bands show the $\mathrm{p}+{ }^{13} \mathrm{~N}$ final-state interaction due to the $33^{-}$state in ${ }^{14} \mathrm{O}$. (b) Inclusive projection $\theta_{p-\mathrm{N}}$ of the data shown in panel (a). (c) Distribution $\theta_{p-\mathrm{N}}$ (full circles with statistical errors) gated by 78 $\operatorname{mrad} \leqslant \sqrt{\theta_{p 1-\mathrm{N}}^{2}+\theta_{p 2-\mathrm{N}}^{2}} \leqslant 88 \mathrm{mrad}$, which corresponds to the $2 \mathrm{p}$ decay of a resonance in ${ }^{15} \mathrm{~F}^{*}$. The solid curve is the simulation of the sequential $2 \mathrm{p}$ decay of ${ }^{15} \mathrm{~F}^{*}$ via the $2^{+}$state in ${ }^{14} \mathrm{O}$ at $E^{*}=6.59 \mathrm{MeV}$ [39]. The fitted parameters of the ${ }^{15} \mathrm{~F}^{*}$ state are $Q_{p}=7.8(2) \mathrm{MeV}$ and $\Gamma=0.4(4) \mathrm{MeV}$. The dotted and dash-dotted curves show similar calculations with assumed ${ }^{15} \mathrm{~F}^{*}$ widths of 1 and $800 \mathrm{keV}$, respectively. The inset shows the probability (as a function of the assumed resonance width) that the simulations match the data. (d) The $\theta_{p 1-N}$ histogram obtained for $\theta_{p 2-\mathrm{N}}>80 \mathrm{mrad}$, which corresponds to $\mathrm{p}+{ }^{13} \mathrm{~N}$ final-state interactions. The solid, dotted, dashed, dash-dotted and dash-dot-dotted curves are simulations of the $1 \mathrm{p}$ resonances in ${ }^{14} \mathrm{O}^{*}$ at $E^{*}$ of $5.173,5.92,6.272,6.59$, and $7.768 \mathrm{MeV}$, respectively [39].

$2 \mathrm{p}$ decay of a high-lying state in ${ }^{16} \mathrm{Ne}$ via ${ }^{15} \mathrm{~F}\left(\frac{1}{2}^{+}\right)$and ${ }^{15} \mathrm{~F}\left(\frac{5}{2}^{+}\right)$with the fitted values of $Q_{2 p}=7.6(2) \mathrm{MeV}$ and $\Gamma_{p}=0.8\left(\begin{array}{c}-0.4 \\ +0.8\end{array}\right) \mathrm{MeV}$. The $P(\Gamma)$ distribution for this state is

TABLE I. The $Q_{p}^{\exp }$ and $\Gamma_{p}^{\exp }$ (in $\mathrm{MeV}$ ) of states observed in ${ }^{15} \mathrm{~F}^{*}$, the assigned spin-parity $J^{\pi}$, the calculated $\operatorname{sps} d p f$ shell-model widths $\Gamma_{p}^{\mathrm{SM}}$ in comparison to the $(s d)^{2}$ shell model [17] and the MCAS [16] predictions. The excitation energies $E_{x}^{15} \mathrm{C}$ of ${ }^{15} \mathrm{C}$ mirror states are from [39].

\begin{tabular}{ccccccccc}
\hline \hline$Q_{p}^{\exp }$ & $\Gamma_{p}^{\exp }$ & $J^{\pi}$ & $\Gamma_{p}^{\mathrm{SM}}$ & $Q_{p}^{[17]}$ & $\Gamma_{p}^{[17]}$ & $Q_{p}^{[16]}$ & $\Gamma_{p}^{[16]}$ & $E_{x}^{15 \mathrm{C}}$ \\
\hline $2.8(1)$ & $0.4(1)$ & $5 / 2^{+}$ & 0.33 & 2.78 & 0.3 & 2.79 & 0.18 & 0.74 \\
$4.9(2)$ & $0.2(2)$ & $1 / 2^{-}$ & 0.09 & 5.49 & 0.005 & 4.63 & 0.055 & 3.10 \\
$6.4(2)$ & $0.2(2)$ & $5 / 2^{-}$ & 0.05 & 6.88 & 0.010 & 5.92 & 0.006 & 4.22 \\
& & $3 / 2^{-}$ & 0.10 & 7.25 & 0.040 & 6.30 & 0.180 & 4.66 \\
$7.8(2)$ & $0.4(4)$ & $3 / 2_{2}^{+}$ & $0.45^{\mathrm{a}}$ & - & - & - & - & 5.83 \\
& & $1 / 2_{2}^{-}$ & $\sim 3^{\mathrm{b}}$ & - & - & - & - & 5.87 \\
& $5 / 2_{2}^{+}$ & $0.3^{\mathrm{a}}$ & 7.75 & 0.4 & - & - & 6.36 \\
\hline
\end{tabular}

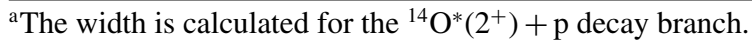

${ }^{\mathrm{b}}$ The width is calculated for the ${ }^{14} \mathrm{O}^{*}\left(1^{-}\right)+\mathrm{p}$ decay branch, which in turn decays into ${ }^{13} \mathrm{~N}+\mathrm{p}+\mathrm{p}$. shown in the inset of Fig. 20(c). The asymmetric shape of $P(\Gamma)$ is due to correlations of the two fit parameters, the level width and the decay branching ratio because larger assumed widths cause smaller admixtures of the $1 / 2^{+}$decay channel. The obtained branching ratios of the $1 / 2^{+}$and $5 / 2^{+}$decay channels are $0.24(8)$ and $0.76(8)$, respectively. The position of the observed ${ }^{16} \mathrm{Ne}^{*}$ state correspond to the $6.1 \mathrm{MeV}$ state in its mirror ${ }^{16} \mathrm{C}$ with $J^{\pi}=\left(2^{+}, 3^{-}, 4^{+}\right)$[40]. We calculated shell-model partial widths of all decay channels for each of these $J^{\pi}$ assignments for the ${ }^{16} \mathrm{Ne}^{*}$ state. The most important ones are shown in Table II. According to these calculations, the only plausible spin-parity of the $7.6 \mathrm{MeV}$ state is $2^{+}$.

TABLE II. Different $\mathbf{J}^{\pi}$ assignment for the $7.6 \mathrm{MeV}$ level in ${ }^{16} \mathrm{Ne}$ and the corresponding partial widths (in $\mathrm{MeV}$ ) for decay into three ${ }^{15} \mathrm{~F}+\mathrm{p}$ channels, calculated in the shell model.

\begin{tabular}{lccc}
\hline \hline$J^{\pi}$ & ${ }^{15} \mathrm{~F}\left(\frac{1}{2}^{+}\right)+\mathrm{p}$ & ${ }^{15} \mathrm{~F}\left(\frac{5}{2}_{1}^{+}\right)+\mathrm{p}$ & ${ }^{15} \mathrm{~F}\left(\frac{1}{2}^{-}\right)+\mathrm{p}$ \\
\hline $2^{+}$ & 0.036 & $>0.37$ & 0.036 \\
$3^{-}$ & 0.007 & $>0.005$ & 0.120 \\
$4^{+}$ & & 1.4 & \\
\hline
\end{tabular}




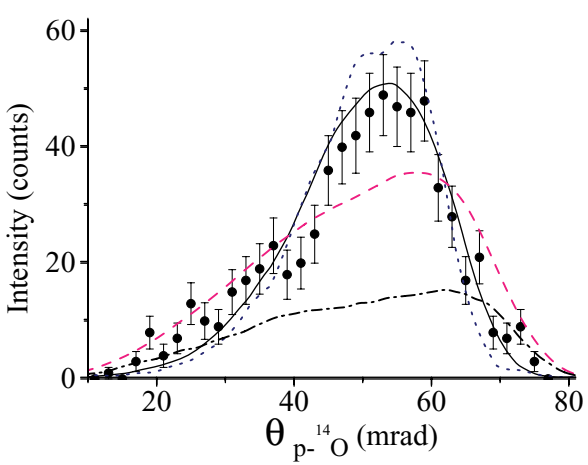

FIG. 22. (Color online) Angular $\mathrm{p}-{ }^{14} \mathrm{O}$ correlations (full circles with statistical uncertainties) obtained from the measured $\mathrm{p}+\mathrm{p}+{ }^{14} \mathrm{O}$ events by selecting the $\rho$ angle within the range from 65 to $80 \mathrm{mrad}$ [see Fig. 14(b)], which corresponds to the first-excited state in ${ }^{16} \mathrm{Ne}$. The solid curve represents the Monte Carlo simulation of the detector response for ${ }^{16} \mathrm{Ne}^{*}\left(2^{+}\right) \rightarrow{ }^{14} \mathrm{O}+\mathrm{p}+\mathrm{p}$ direct $2 \mathrm{p}$ decay with the fitted values $Q_{2 p}=3.2(2) \mathrm{MeV}$ and $\Gamma=0.2(2) \mathrm{MeV}$. The dotted line presents the same calculation but assuming a different $2 \mathrm{p}$-decay mechanism, namely a sequential proton emission via ${ }^{15} \mathrm{~F}$ g.s. The dashed line is a simulation of a hypothetical direct $2 p$ decay of the $0^{+}$state with $Q_{2 p}=3.5 \mathrm{MeV}$ and $\Gamma=1 \mathrm{MeV}$ reported in Ref. [41]. The dash-dotted curve indicates the background contribution scaled from the $7.6 \mathrm{MeV}$ state data.

In addition to the previously discussed two states in ${ }^{16} \mathrm{Ne}$, we found an evidence for the first-excited state $2^{+}$ in ${ }^{16} \mathrm{Ne}$ by inspecting the $\rho$-angular distribution shown in Fig. 14(b). The intermediate part of the $\rho$ distribution cannot be reproduced by only the ground $1.4 \mathrm{MeV}$ and the excited 7.6 MeV states. However, a quantitative description of the data can be achieved by adding an excited state in ${ }^{16} \mathrm{Ne}$ with the fitted values of $2 \mathrm{p}$-decay energy $Q_{2 p}=3.2(2) \mathrm{MeV}$ and width $\Gamma_{p}=0.2(2) \mathrm{MeV}$. These values were obtained by fitting the corresponding angular $\mathrm{p}^{-1}{ }^{14} \mathrm{O}$ correlations as illustrated in Fig. 22. One may see that both direct and sequential 2 p-decay mechanisms produce very similar distributions, which we cannot distinguish with the present experimental uncertainties. The obtained values are in quantitative agreement with the previous measurements reporting for $2^{+} Q_{2 p}=3.09$ (7) $\mathrm{MeV}$ [32] and provide the first evaluation of its width which is less than $0.2 \mathrm{MeV}$. The measured width value is crucial for assigning spin-parity of this state. In the article [41] a first excited state in ${ }^{16} \mathrm{Ne}$ was found at $Q_{2 p}=3.5 \mathrm{MeV}$, and it was identified as a second $0^{+}$rather than $2^{+}$. The positions of these two states are quite close, however, their widths should be 1 and $0.01 \mathrm{MeV}$, respectively, according to the theoretical predictions [33]. Our data are consistent with spin-parity of $2^{+}$ only. The level and decay scheme of the ${ }^{16} \mathrm{Ne}$ precursor states derived from our measurements are included in Fig. 23.

Summarizing this chapter, we measured $1 p$ and $2 p$ decays of excited states in ${ }^{15} \mathrm{~F}$ and ${ }^{16} \mathrm{Ne}$ that can be explained as valence protons coupled to excited-core states, which are, in turn, proton resonances. For the $Q_{p}=4.9$ and $6.4 \mathrm{MeV}$ states in ${ }^{15} \mathrm{~F}$, the proton energies of such a configuration are too small and the decay into excited-core daughter states is suppressed by the Coulomb barrier. In the cases of ${ }^{15} \mathrm{~F}^{*}$ $\left(Q_{p}=7.8 \mathrm{MeV}\right)$ and ${ }^{16} \mathrm{Ne}^{*}\left(Q_{p}=7.6 \mathrm{MeV}\right)$ these energies

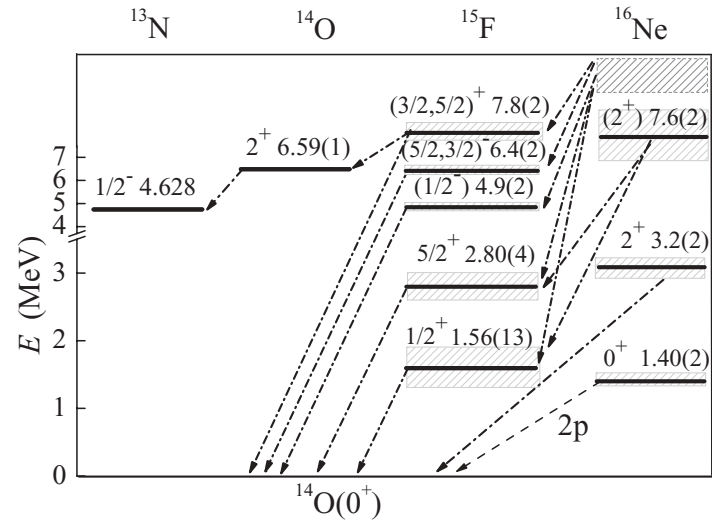

FIG. 23. Level scheme of all states observed in our experiment. Single-proton $1 \mathrm{p}$ decays of the states in ${ }^{16} \mathrm{Ne},{ }^{15} \mathrm{~F}$, and ${ }^{14} \mathrm{O}$ are shown by dash-dotted arrows. The corresponding decay energies are shown relative to the respective ${ }^{14} \mathrm{O}+1 \mathrm{p}(2 \mathrm{p})$ thresholds. A $2 p$ decay of the ${ }^{16} \mathrm{Ne}$ g.s. is illustrated by the dashed arrow. The hatched area indicates the high-energy continuum in ${ }^{16} \mathrm{Ne}$.

are larger and their decays into excited-core daughter states dominate. In all cases the relation $\Gamma_{p} \leqslant Q_{p}$ holds, which means that observed nuclear configurations beyond the proton drip line are relatively stable.

\section{SUMMARY AND OUTLOOK}

The described method of in-flight $1 \mathrm{p}$ - and $2 \mathrm{p}$-decay measurements by tracking all decay products is a powerful technique in studies of $1 \mathrm{p}$ - and $2 \mathrm{p}$-unbound nuclei, which has several advantages over the conventional missing-mass and invariant-mass methods. In particular,

(i) Measurements of the total energies of the protons are not required. The method allows to measure the decay energies and widths of the $1 \mathrm{p}$ - and $2 \mathrm{p}$-precursor states by using only angular correlations between decay products. Three-body angular correlations provide information on nuclear structure and decay mechanisms.

(ii) The method is ideally suited for low-intensity beams of exotic nuclei near the proton-drip line. Thick targets (up to several $\mathrm{g} / \mathrm{cm}^{2}$ ) and low-quality beams can be used without loosing too much precision in measuring the angles between the decay products, which is crucial for determining the resonance energies and widths. A relatively high precision of the measured decay energies of 50-200 keV at incident energies of $500 A \mathrm{MeV}$ was demonstrated.

(iii) Large registration efficiencies (of about 30-70\% for three-particle events) allow studies of multiparticle decay branches.

In a forthcoming article, we will explain in detail how 2 p-decay lifetimes in the range from nanoseconds to femtoseconds can be measured with this technique, as was demonstrated for the $2 \mathrm{p}$ decay of the ${ }^{19} \mathrm{Mg}$ g.s. [3]. Since this type of measurement requires only a rather simple setup and can be applied to proton-unbound nuclei with very small production cross sections, many more nuclei are expected to be studied 
with this method in the future (e.g., see some theoretically predicted cases in Refs. [20,42]).

\section{ACKNOWLEDGMENTS}

The authors are very much indebted to $\mathrm{M}$. Pohl and his co-workers of the DPNC, Université de Genève for developing the microstrip detectors. We are particularly grateful for the valuable contribution to this project by E. Cortina. We thank A. Brünle, K. H. Behr, W. Hüller, C. Karagiannis, A. KelićHeil, A. Kiseleva, R. Raabe, and O. Tarasov for their help in preparation of the experiment. This work has been supported by Contract EURONS Nos. EC-I3 and FPA2006-13807-C0201, FPA2007-63074 (MEC, Spain), the INTAS Grant No. 03-54-6545. L.V.G. is supported by FAIR-Russia Research Center grant, Russian Foundation for Basic Research via Grant Nos. RFBR 08-02-00892 and 08-02-00089-a, and Russian Ministry of Industry and Science Grant No. NSh-7235.2010.2. N.K.T. acknowledges support from the UK Grant No. STFC ST/F012012/1, E.L. is supported by the LOEWE program of the State of Hessen (Helmholtz International Center for FAIR), Germany.
[1] M. Pfützner et al., Eur. Phys. J. A 14, 279 (2002); J. Giovinazzo et al., Phys. Rev. Lett. 89, 102501 (2002).

[2] B. Blank et al., Phys. Rev. Lett. 94, 232501 (2005).

[3] I. Mukha et al., Phys. Rev. Lett. 99, 182501 (2007).

[4] I. Mukha et al., Nature (London) 439, 298 (2006).

[5] L. V. Grigorenko, R. C. Johnson, I. G. Mukha, I. J. Thompson, and M. V. Zhukov, Phys. Rev. Lett. 85, 22 (2000).

[6] L. V. Grigorenko, R. C. Johnson, I. G. Mukha, I. J. Thompson, and M. V. Zhukov, Phys. Rev. C 64, 054002 (2001).

[7] L. V. Grigorenko, I. G. Mukha, and M. V. Zhukov, Nucl. Phys. A 713, 372 (2003); 740, 401(E) (2004).

[8] H. Schatz et al., Phys. Rep. 294, 167 (1998).

[9] A. Wöhr et al., Nucl. Phys. A 742, 349 (2004).

[10] L. V. Grigorenko and M. V. Zhukov, Phys. Rev. C 72, 015803 (2005).

[11] I. Mukha et al., Phys. Rev. C 79, 061301(R) (2009).

[12] A. Lepine-Szily et al., Nucl. Phys. A 734, 331 (2004).

[13] W. A. Peters et al., Phys. Rev. C 68, 034607 (2003).

[14] V. Z. Goldberg et al., Phys. Rev. C 69, 031302(R) (2004).

[15] K. Markenroth et al., Phys. Rev. C 62, 034308 (2000).

[16] L. Canton, G. Pisent, J. P. Svenne, K. Amos, and S. Karataglidis, Phys. Rev. Lett. 96, 072502 (2006); 99, 089202 (2007).

[17] H. T. Fortune and R. Sherr, Phys. Rev. Lett. 99, 089201 (2007); H. T. Fortune, Phys. Rev. C 74, 054310 (2006).

[18] I. Mukha and G. Schrieder, Nucl. Phys. A 690, 280c (2001).

[19] I. Mukha, Phys. At. Nucl. 66, 1519 (2003).

[20] L. V. Grigorenko and M. V. Zhukov, Phys. Rev. C 68, 054005 (2003).

[21] I. Mukha et al., Phys. Rev. C 77, 061303(R) (2008).

[22] H. Geissel et al., Nucl. Instrum. Methods Phys. Res., Sect. B 70, 286 (1992).

[23] M. Stanoiu et al., Nucl. Instrum. Methods Phys. Res., Sect. B 266, 4625 (2008).
[24] B. Alpat et al., Nucl. Instrum. Methods Phys. Res., Sect. A 540, 121 (2005).

[25] V. Karimki, T. Lampn, and F.-P. Schilling, IEEE Trans. Nucl. Sci. 53(6), 3830 (2006).

[26] I. Mukha, Eur. Phys. J. A 42, 421 (2009).

[27] J. Hoffmann, N. Kurz, and W. Ott, GSI Scientific Report 2006, p. 216.

[28] V. I. Goldansky, Nucl. Phys. 19, 482 (1960).

[29] A. I. Baz', V. I. Goldansky, V. Z. Goldberg, and Ya. B. Zeldovich, Light and Intermediate Nuclei Near the Border of Nuclear Stablity (Nauka, Moscow, 1972).

[30] A. M. Lane and R. G. Thomas, Rev. Mod. Phys. 30, 257 (1958).

[31] C. J. Woodward, R. E. Tribble, and D. M. Tanner, Phys. Rev. C 27, 27 (1983).

[32] G. J. KeKelis, M. S. Zisman, D. K. Scott, R. Jahn, D. J. Vieira, J. Cerny, and F. Ajzenberg-Selove, Phys. Rev. C 17, 1929 (1978).

[33] L. V. Grigorenko, I. G. Mukha, I. J. Thompson, and M. V. Zhukov, Phys. Rev. Lett. 88, 042502 (2002).

[34] GEANT-detector simulation tool, CERN software library, [http://wwwasd.web.cern.ch/wwwasd/geant].

[35] W. T. Eadie et al., Statistical Methods in Experimental Physics (North-Holland, Amsterdam, 1971).

[36] W. Benenson et al., Phys. Lett. B 58, 46 (1975).

[37] B. A. Brown, Prog. Part. Nucl. Phys. 47, 517 (2001).

[38] B. A. Brown and W. D. M. Rae, NUSHELL@MSU, MSU-NSCL Report, 2007 (unpublished).

[39] F. Ajzenberg-Selove, J. H. Kelley, and C. D. Nesaraja, Nucl. Phys. A 523, 1 (1991).

[40] D. R. Tilley, H. R. Weller, and C. M. Cheves, Nucl. Phys. A 564, 1 (1993).

[41] K. Föhl et al., Phys. Rev. Lett. 79, 3849 (1997).

[42] L. V. Grigorenko, I. G. Mukha, and M. V. Zhukov, Nucl. Phys. A 714, 425 (2003). 